\title{
A Semi-Lagrangian Approach for Natural Gas Storage Valuation and Optimal Operation
}

\author{
Zhuliang Chen* Peter A. Forsyth ${ }^{\dagger}$
}

October 2, 2006

\begin{abstract}
The valuation of a gas storage facility is characterized as a stochastic control problem, resulting in a Hamilton-Jacobi-Bellman (HJB) equation. In this paper, we present a semi-Lagrangian method for solving the HJB equation for a typical gas storage valuation problem. The method is able to handle a wide class of spot price models that exhibit mean-reverting, seasonality dynamics and price jumps. We develop fully implicit and Crank-Nicolson timestepping schemes based on a semi-Lagrangian approach and prove the convergence of fully implicit timestepping to the viscosity solution of the HJB equation. We show that fully implicit timestepping is equivalent to a discrete control strategy, which allows for a convenient interpretation of the optimal controls. The semi-Lagrangian approach avoids the nonlinear iterations required by an implicit finite difference method without requiring additional cost. Numerical experiments are presented for several variants of the basic scheme.

Keywords: gas storage, semi-Lagrangian, HJB equation, stochastic control, viscosity solution

Acknowledgment: This work was supported by the Natural Sciences and Engineering Research Council of Canada, and by a Morgan Stanley Equity Market Microstructure Research Grant. The views expressed herein are solely those of the authors, and not those of any other person or entity, including Morgan Stanley.
\end{abstract}

\section{Introduction}

Similar to other commodities such as fuel and electricity, natural gas prices exhibit seasonality dynamics due to fluctuations in demand [28]. As such, natural gas storage facilities are constructed to provide a cushion for such fluctuations by releasing natural gas in storage in seasons with high demand.

Recently, several authors $[1,32,35,36,25,11,8]$ have discussed the no-arbitrage value of natural gas storage facilities (or, equivalently, the values of gas storage contracts for leasing the storage facilities). The value of a gas storage facility can be regarded as the maximum expected revenues that the operator of the facility can obtain by optimally operating the facility, that is, "buying

\footnotetext{
* David R. Cheriton School of Computer Science, University of Waterloo, Waterloo ON, Canada N2L 3G1 e-mail: z4chen@uwaterloo.ca

${ }^{\dagger}$ David R. Cheriton School of Computer Science, University of Waterloo, Waterloo ON, Canada N2L 3G1 e-mail: paforsyt@uwaterloo.ca
} 
low" and "selling high". As a result, the valuation of gas storage facilities is characterized as a stochastic control problem.

Two contrasting approaches have been used to solve this problem numerically: partial differential equation (PDE) based approaches and simulation based methods. Assuming that the control is of bang-bang type (that is, the control only takes values from a finite set), simulation based methods $[11,8]$ can be used to directly solve the stochastic control problem. It is known that such methods have difficulty achieving high accuracy. Furthermore, if the control is not of bang-bang type, such methods will have to approximate the control as piece-wise constant, which will substantially increase the computational cost. See [36, 25] and [33] for descriptions of control problems which are not of the bang-bang type for storage swing options and valuation of electricity power plants.

As shown in $[27,21]$, the value of a stochastic control problem is identical to the viscosity solution of a Hamilton-Jacobi-Bellman (HJB) equation. As a result, the value of a gas storage contract can be computed by solving the corresponding HJB equation using PDE based approaches. In general, the solution to the HJB equation may not be unique. As noted in $[22,2]$, it is important to ensure that a numerical scheme converges to the viscosity solution of the equation, which is the appropriate value of the corresponding stochastic control problem.

In [32], an explicit finite difference scheme is used to price gas storage contracts. As is well known, explicit timestepping suffers from timestep restrictions due to stability considerations. Alternatively, the authors of [22] present implicit finite difference schemes, which eliminate the timestep restriction, for solving general controlled HJB equations. However, this scheme requires solution of nonlinear discretized algebraic equations using a Newton-like iteration at each timestep. Reference [22] introduces another type of implicit scheme that approximates the control as piecewise constant to avoid the need for solving nonlinear equations at the expense of solving a number of linear problems at each timestep. Similar to the simulation based methods, if the control is not of bang-bang type, such schemes will be computationally expensive. In [35], a finite element semiLagrangian scheme is developed to solve an HJB equation with no bang-bang control for certain gas storage contracts. In [36, 25], a wavelet method coupled with a semi-Lagrangian approach is used to solve the gas storage HJB equation. While the wavelet method shows promise, it is difficult to obtain a rigorous proof of convergence to the viscosity solution.

In this paper, following [32], we formulate the value of a gas storage facility as a two-dimensional HJB equation. For the particular model selected, the controls can be shown to be of the bangbang type [32]. Since the equation is convection dominated, that is, the equation has no diffusion in one of the coordinate directions, it is well known to be difficult to solve numerically. Initially introduced by $[18,29]$ for atmospheric and weather numerical predictions, semi-Lagrangian schemes can effectually reduce the numerical problems arising for convection dominated equations. In [15], a semi-Lagrangian method is applied to price continuously observed American Asian options.

We apply the semi-Lagrangian approach to solve the HJB equation for gas storage contracts in this paper. We consider only fairly simple forms for the spot price model, but our method can easily generalize to more complex spot price models. To illustrate this, we also include numerical experiments using a process that incorporates price jumps.

The semi-Lagrangian discretizations require solution of discrete local optimization problems in order to determine optimal control values. Although the controls for the exact solution of the pricing HJB equation are of bang-bang type, the solutions of the discrete optimization problems may allow controls which are not possible controls for the exact solution. Consequently, two methods are used 
to solve the optimization problems: the bang-bang method that considers only bang-bang controls as appearing in the exact solution, and the no bang-bang method that actually solves the discrete problem, and hence allows any admissible controls.

The main results of this paper are summarized as follows:

- We demonstrate that a semi-Lagrangian method can be used to price gas storage contracts and obtain optimal control strategies under a wide range of spot price models that exhibit mean-reverting, seasonality dynamics and price jumps. A semi-Lagrangian method reduces the problem to solving a decoupled set of one-dimensional nonlinear discrete PDEs at each timestep. This makes implementation of this method very straightforward in a software library which is capable of pricing path-dependent options [39].

- We show that the fully implicit, semi-Lagrangian discretization is algebraically identical to a discretization derived from a discrete control strategy. This means that the fully implicit, semi-Lagrangian discretization satisfies discrete no-arbitrage jump conditions, which is an important practical consideration.

- We show that, as opposed to implicit finite difference discretizations, the semi-Lagrangian discretizations avoid the need for nonlinear iterations at each timestep. Moreover, the discretizations do not introduce extra costs, as required by the piece-wise constant control method [22].

- We prove that the fully implicit, semi-Lagrangian discretization converges to the viscosity solution of the HJB equation by verifying the stability, consistency and monotonicity of the scheme and using the basic results in $[7,2]$. We pay particular attention to the boundary conditions and properties of the admissible controls on the boundary of the computational domain, to ensure that the conditions required for convergence to the viscosity solution are met.

- Numerical results show that fully implicit and Crank-Nicolson timestepping schemes using the semi-Lagrangian method converge to the same solution. In the case of fully implicit timestepping, the no bang-bang method is superior to the bang-bang method, since the no bang-bang method converges smoothly at a first-order rate for some cases that cause numerical difficulties for the bang-bang method. Numerical results also indicate that CrankNicolson timestepping does not appear to converge at a higher than first order rate, and hence fully implicit timestepping is probably a better choice since it guarantees convergence to the viscosity solution. Fully implicit timestepping is also straightforward to implement.

\section{Gas Storage Equation}

This section defines the natural gas storage problem and corresponding partial differential equation (PDE) for the value of a gas storage contract. The section is arranged as follows: first, we define some notation for the problem; we then present two mean-reverting models for the natural gas spot price. For both models, we give a general PDE for the value of natural gas storage contract. Finally, we completely specify the gas storage problem by providing the boundary conditions for the PDE. 


\subsection{Problem notation}

We use the following notation for the natural gas storage problem:

- $P$ : the current spot price per unit of natural gas.

- $I$ : current amount of working gas inventory. We assume that $I$ can be any value lying within the domain $\left[0, I_{\max }\right]$.

- $\hat{V}(P, I, t)$ : value of the natural gas storage facility (e.g., the leasing rate of the facility) with respect to natural gas price $P$ and inventory level $I$ at time $t$.

- $T$ : expiry time of the contract.

- $c$ : control variable that represents the rate of producing gas from or injecting gas into the gas storage ( $c>0$ represents production, $c<0$ represents injection). If $c=0$ then no operation is performed on the storage.

- $c_{\max }(I)$ : the maximum rate at which gas can be released from storage as a function of inventory levels, $c_{\max }(I)>0$. We use the expression in [32]

$$
c_{\max }(I)=k_{1} \sqrt{I}
$$

where $k_{1}$ is a positive constant. This implies $c_{\max }(0)=0$ with the physical meaning that no gas can be produced if the gas storage is empty.

- $c_{\min }(I):\left|c_{\min }(I)\right|$ is the maximum rate at which gas can be injected into storage as a function of inventory levels. Note that $c_{\min }(I)<0$, with our sign convention that $c>0$ represents production. We use the expression from [32]

$$
c_{\min }(I)=-k_{2} \sqrt{\frac{1}{I+k_{3}}-\frac{1}{k_{4}}},
$$

where $k_{2}, k_{3}$ and $k_{4}$ are positive constants. $k_{1}, k_{2}, k_{3}$ satisfy the constraint $c_{\min }\left(I_{\max }\right)=0$, which means that no gas injection is possible if the gas storage is full. Equation (2.2) implies that

$$
\left|c_{\min }(I)\right| \leq \mid \text { const. } \sqrt{I_{\max }-I} \mid ; I<I_{\max }, I \rightarrow I_{\max } .
$$

- $a(I, c)$ : the rate of gas loss given a gas production rate of $c$, when $I$ units are currently in storage. In general, the change in gas inventory satisfies

$$
\frac{d I}{d t}=-(c+a(I, c))
$$

where usually $a(I, c) \geq 0$. We use the model in [32]

$$
a(I, c)=a(c)=\left\{\begin{array}{ll}
0 & \text { if } c \geq 0, \text { (producing gas) } \\
k_{5} & \text { if } c<0 . \text { (injecting gas) }
\end{array},\right.
$$

where $k_{5}>0$ is a constant. 
Note that if we are using a control $c$ satisfying $-k_{5}<c<0$, that is, injecting at a rate smaller than the rate of gas loss, then equation (2.5) implies that $c+a(c)>0$. According to equation (2.4), this means that injecting natural gas into the gas storage decreases the gas inventory, which is is unreasonable. Consequently, we further require that control $c$ satisfies the constraint $c \in\left[c_{\min }(I),-k_{5}\right] \cup\left[0, c_{\max }(I)\right]$ so that

$$
c+a(c) \leq 0 \quad \text { if } c<0 \text { (injecting gas). }
$$

In other words, the operator of the gas storage facility is not allowed to inject and at the same time decrease the gas inventory. We point out that the constraint on the control also makes the boundary conditions well defined (this will be discussed in more detail in a subsequent section). For future reference, given any $I \in\left[0, I_{\max }\right]$, we define the set $C(I)$ as

$$
C(I)=\left[c_{\min }(I),-k_{5}\right] \cup\left[0, c_{\max }(I)\right]
$$

where we adopt the convention that $[\alpha, \beta]=\emptyset$ if $\alpha>\beta$.

\subsection{Natural gas spot price models}

In this subsection, we present two one-factor mean-reverting processes for natural gas spot price. In the following we will refer to these processes as pure mean-reverting process and geometric meanreverting process, respectively. Both of these processes are able to capture the mean-reverting and seasonality effects of natural gas spot prices. However, there is a certain amount of controversy surrounding the precise form of a reasonable natural gas spot price model [28]. The numerical methods that we derive in this paper easily generalize to more complex spot price models.

\section{Pure mean-reverting process}

We note that the gas storage problem is a real options valuation problem [17, 34]. Hence, the usual derivation of the pricing equation starts with the actual spot price process, and a hedging argument generates a PDE with a risk adjusted drift term [34]. We refer interested readers to [34, 28] for details. We will take a short cut here and simply consider the risk adjusted price process, since this is the process which appears in the final PDE.

In this model, the risk adjusted gas spot price follows a mean-reverting stochastic process with the equilibrium price varying over time to produce a seasonality effect. The risk adjusted (or risk neutral) gas spot price is modeled by a stochastic differential equation given by

$$
\begin{aligned}
d P & =\alpha(K(t)-P) d t+\sigma P d Z \\
K(t) & =K_{0}+\beta_{S A} \sin \left(4 \pi\left(t-t_{S A}\right)\right),
\end{aligned}
$$

where

- $\alpha>0$ is the mean-reverting rate,

- $K(t) \geq 0$ is the long-term equilibrium price that incorporates seasonality,

- $\sigma$ is the volatility,

- $d Z$ is an increment of the standard Gauss-Wiener process, 
- $K_{0} \geq 0$ is the equilibrium price without seasonality effect,

- $\beta_{S A}$ is the semiannual seasonality parameter,

- $t_{S A}$ is the seasonality centering parameters, representing the time of semiannual peak of equilibrium price in summer and winter.

According to equation (2.9), the equilibrium price $K(t)$ is a periodic function with period $1 / 2$. This models the seasonal evolution of the annual equilibrium price, e.g., $K(t)$ exhibits two peaks annually, respectively corresponding to high natural gas spot prices in summer and winter.

\section{Geometric mean-reverting process}

In this model, the potential natural gas price is modeled by a stochastic differential equation given by

$$
d P=\alpha(\log K(t)-\log P) P d t+\sigma P d Z
$$

where $K(t)$ is given by equation (2.9). The parameters for equation (2.10) are the same as for the pure mean reverting model.

Let $x=\log P$. From equation (2.10), using Ito's lemma gives

$$
d x=\alpha\left(\log K(t)-\frac{\sigma^{2}}{2 \alpha}-x\right) d t+\sigma d Z .
$$

Equation (2.11) implies that logarithm of natural gas spot price follows a mean-reverting stochastic process with the equilibrium price equal to $\log K(t)-\frac{\sigma^{2}}{2 \alpha}$. Thus, from equation (2.9), the equilibrium natural gas spot price, which is approximately equal to $K(t)$, shows a seasonality effect.

We can write the risk adjusted gas spot price processes (2.8) and (2.10) more compactly using a general stochastic process

$$
d P=\mu(P, t) d t+\sigma P d Z
$$

where

$$
\mu(P, t)=\left\{\begin{array}{cc}
\alpha(K(t)-P) & \text { for pure mean-reverting process }(2.8), \\
\alpha(\log K(t)-\log P) P & \text { for geometric mean-reverting process }(2.10) .
\end{array}\right.
$$

Note that $\mu(P, t)$ is also the drift of the forward price process, and hence the parameters of the drift model can be obtained by calibration to forward prices.

\subsection{The PDE for natural gas storage facilities}

For a financial contract such as the natural gas storage contract, the payoff is given at the maturity $t=T$. In order to compute the value of the contract today, we need to solve the pricing PDE backwards in time from $t=T$ to $t=0$. Let $\tau=T-t$ denote the current time-to-maturity. For ease of exposition, we will write our PDE in terms of $\tau$ so that we will solve the pricing PDE from $\tau=0$ to $\tau=T$. Let $V(P, I, \tau)$ denote the value of a natural gas storage facility as a function of $(P, I, \tau)$. In terms of the facility value $\hat{V}(P, I, t)$ at forward times with respect to $t$, we have the identity $V(P, I, \tau)=\hat{V}(P, I, T-\tau)=\hat{V}(P, I, t)$. 
Based on the standard hedging arguments in the financial valuation literature [32], the value of a natural gas storage facility in terms of $\tau$, assuming that the risk adjusted natural gas spot price follows the stochastic process defined in (2.12) is given by

$$
V_{\tau}=\frac{1}{2} \sigma^{2} P^{2} V_{P P}+\mu(P, t) V_{P}+\max _{c \in C(I)}\left\{(c-a(c)) P-(c+a(c)) V_{I}\right\}-r V,
$$

where $C(I)$ is given in (2.7) and $r$ is the continuously compounded risk-free interest rate. The nonlinear PDE (2.14) is known as the Hamilton-Jacobi-Bellman (HJB) equation. In equation (2.14), the term $(c-a(c)) P$ represents the instantaneous rate of revenue obtained by producing natural gas from $(c>0)$ or injecting gas into $(c<0)$ the gas storage facility. The term $-(c+a(c)) V_{I}$ results from the rate of inventory change (recall equation $(2.4)$ ).

\subsection{Boundary conditions for the pricing PDE}

In order to completely specify the gas storage problem, we need to provide boundary conditions. We will proceed in an informal fashion here to derive plausible boundary conditions. These boundary conditions will be justified in a more rigorous fashion in Section 4 .

A number of terminal boundary conditions can be used. Note that since we will be solving backwards in time, the terminal state occurs at $\tau=0$. Typical examples include

- A zero payoff, as suggested by [32]: $V(P, I, \tau=0)=0$.

- A non-negative payoff obtained by selling all the leftover gas in the storage at the maximum rate, that is, $V(P, I, \tau=0)$ is the solution $\bar{V}$ to the PDE obtained by fixing control $c=$ $c_{\max }(I)$ in PDE (2.14) and solving the resulting PDE backwards from $\tau=0$ to $\tau=\infty$ with $\bar{V}(P, I, \tau=0)=0$. We then specify $V(P, I, \tau=0)=\bar{V}(P, I, \tau=\infty)$.

- The penalty payoff introduced by [11]:

$$
V(P, I, \tau=0)=\text { const. } \cdot P \cdot \min (I(t=T)-I(t=0), 0),
$$

where const. $>0$. This has the meaning that a penalty will be charged if the gas inventory in storage when the facility is returned is less than the gas inventory at the inception of the contract.

The domain for the PDE (2.14) is $P \times I \in[0, \infty] \times\left[0, I_{\max }\right]$. For computational purposes, we need to solve the PDE in a finite computational domain $\left[0, P_{\max }\right] \times\left[0, I_{\max }\right]$.

As $I \rightarrow 0$, from equations $(2.1-2.7)$ we have that

$$
c+a(c) \leq 0 ; \forall c \in C(I), I \rightarrow 0 .
$$

Hence the characteristics are outgoing in the $I$ direction at $I=0$, and we simply solve the PDE along the $I=0$ boundary, no further information is needed. Condition (2.16) has the interpretation that gas cannot be produced from a facility which is empty.

Similarly, as $I \rightarrow I_{\max }$, equations (2.1-2.7) imply that

$$
c+a(c) \geq 0 \quad ; \quad \forall c \in C(I), I \rightarrow I_{\max },
$$


which again means that the characteristics are outgoing in the $I$ direction at $I=I_{\max }$. Consequently, we simply solve the PDE along the $I=I_{\max }$ boundary, no further information is needed. Condition (2.17) has the interpretation that gas cannot be injected into the storage facility when it reaches full capacity.

Taking the limit of equation (2.14) as $P \rightarrow 0$, we obtain

$$
V_{\tau}=\left.\mu(P, t)\right|_{P \rightarrow 0} V_{P}+\max _{c \in C(I)}\left\{-(c+a(c)) V_{I}\right\}-r V
$$

where $\left.\mu(P, t)\right|_{P \rightarrow 0}$ satisfies $\left.\mu(P, t)\right|_{P \rightarrow 0}=\alpha K(t)$ or $\left.\mu(P, t)\right|_{P \rightarrow 0}=0$ respectively for the case when the risk adjusted gas spot price follows a pure mean-reverting process (2.8) and a geometric mean-reverting process (2.10). Since $\left.\mu(P, t)\right|_{P \rightarrow 0} \geq 0$, we can solve (2.18) without requiring additional boundary conditions, as we do not need information from outside the computational domain $\left[0, P_{\max }\right]$.

As $P \rightarrow P_{\max }$, we apply the commonly used boundary condition $V_{P P} \rightarrow 0$ [38], so that the pricing equation (2.14) becomes

$$
V_{\tau}=\left.\mu(P, t)\right|_{P \rightarrow P_{\max }} V_{P}+\max _{c \in C(I)}\left\{(c-a(c)) P-(c+a(c)) V_{I}\right\}-r V \quad ; \quad P \rightarrow P_{\max },
$$

where $\left.\mu(P, t)\right|_{P \rightarrow P_{\max }}$ satisfies $\left.\mu(P, t)\right|_{P \rightarrow P_{\max }}=\alpha\left(K(t)-P_{\max }\right)$ or $\left.\mu(P, t)\right|_{P \rightarrow P_{\max }}=\alpha(\log K(t)-$ $\left.\log P_{\max }\right)$ for process $(2.8)$ and $(2.10)$, respectively. We will always choose $P_{\max } \gg K(t)$, hence equation $(2.19)$ can be solved at $P=P_{\max }$ without additional information.

Note that for classical solutions, additional conditions are required on $\sigma(P)$ as $P \rightarrow 0, P_{\max }$ [26]. However, these conditions are not required for viscosity solutions, as discussed in Section 4.

\section{Semi-Lagrangian Discretization}

A semi-Lagrangian approach is introduced in [15] for pricing continuously observed American Asian options under jump diffusion. In this section, we extend the semi-Lagrangian method from [15] to solve the HJB equation (2.14) that involves optimal control. The main idea used to construct a semi-Lagrangian discretization of the PDE (2.14) is to integrate the PDE along a semi-Lagrangian trajectory (defined below). Various semi-Lagrangian discretizations can be obtained by evaluating the resulting integrals using different numerical integration methods: for example, using the rectangular rule provides a fully implicit timestepping scheme, while using the trapezoidal rule gives a Crank-Nicolson timestepping scheme.

This section is arranged as follows: we first present an intuitive idea for developing a semiLagrangian discretization for equation (2.14). We then present both a fully implicit and a CrankNicolson timestepping scheme based on this idea. We will show that the fully implicit semiLagrangian scheme is identical to a scheme derived based on a purely physical reasoning, described in Appendix B. This ensures that the fully implicit semi-Lagrangian scheme satisfies discrete noarbitrage jump conditions. At the end of this section, we reformulate the discrete equations resulting from the semi-Lagrangian discretization into a matrix form as an aid in the analysis presented in later sections.

Prior to presenting the timestepping schemes, we introduce the following notation. We use an unequally spaced grid in $P$ direction for the PDE discretization, represented by $\left[P_{1}, P_{2}, \ldots, P_{i_{\max }}\right]$. Similarly, we use an unequally spaced grid in the $I$ direction denoted by $\left[I_{1}, I_{2}, \ldots, I_{j_{\max }}\right]$. We 
denote by $0=\tau^{0}<, \ldots,<\tau^{N}=T$ the discrete timesteps used to discretize the PDE (2.14). Let $\Delta \tau^{n}=\tau^{n+1}-\tau^{n}$. Let $V\left(P_{i}, I_{j}, \tau^{n}\right)$ denote the exact solution of equation (2.14) when the natural gas spot price resides at node $P_{i}$, the gas inventory at node $I_{j}$ and discrete time at $\tau^{n}$. Let $V_{i, j}^{n}$ denote an approximation of the exact solution $V\left(P_{i}, I_{j}, \tau^{n}\right)$. Let $\mathcal{L}$ be a differential operator represented by

$$
\mathcal{L} V=\frac{1}{2} \sigma^{2} P^{2} V_{P P}+\mu(P, t) V_{P}-r V .
$$

Using the differential operator (3.1), we can rewrite natural gas storage pricing PDE (2.14) as

$$
\min _{c \in C(I)}\left\{V_{\tau}+(c+a(c)) V_{I}-(c-a(c)) P-\mathcal{L} V\right\}=0 .
$$

We use standard finite difference methods to discretize the operator $\mathcal{L} V$. Let $\left(\mathcal{L}_{h} V\right)_{i, j}^{n}$ denote the discrete value of the differential operator (3.1) at node $\left(P_{i}, I_{j}, \tau^{n}\right)$. The operator (3.1) can be discretized using central, forward, or backward differencing in the $P, I$ directions to give

$$
\left(\mathcal{L}_{h} V\right)_{i, j}^{n}=\alpha_{i}^{n} V_{i-1, j}^{n}+\beta_{i}^{n} V_{i+1, j}^{n}-\left(\alpha_{i}^{n}+\beta_{i}^{n}+r\right) V_{i, j}^{n},
$$

where $\alpha_{i}^{n}$ and $\beta_{i}^{n}$ are determined using the algorithm in Appendix A. The algorithm guarantees $\alpha_{i}^{n}$ and $\beta_{i}^{n}$ satisfy the following positive coefficient condition:

$$
\alpha_{i}^{n} \geq 0 \quad ; \quad \beta_{i}^{n} \geq 0 \quad i=1, \ldots, i_{\max } ; j=1, \ldots, j_{\max } ; n=1, \ldots, N .
$$

As we will demonstrate in Section 4, the positive coefficient property (3.4) is a sufficient condition to ensure convergence of a semi-Lagrangian fully implicit timestepping scheme to the viscosity solution of the HJB equation (2.14).

Now we give the intuition for developing the semi-Lagrangian discretization schemes. Let us consider a path (or a semi-Lagrangian trajectory) $\mathcal{I}(\tau)$ that follows the ODE

$$
\frac{d \mathcal{I}}{d \tau}=c+a(c)
$$

According to (3.5), we can write the term $V_{\tau}+(c+a(c)) V_{I}$ in (3.2) in the form of a Lagrangian directional derivative

$$
\frac{D V}{D \tau}=\frac{\partial V}{\partial \tau}+\frac{\partial V}{\partial I} \frac{d \mathcal{I}}{d \tau}
$$

Then equation (3.2) can be rewritten as

$$
\min _{c \in C(I)}\left\{\frac{D V}{D \tau}-(c-a(c)) P-\mathcal{L} V\right\}=0 .
$$

Let us analyze (3.5-3.7) from a discrete point of view, that is, consider discrete grid points and discrete times. Let $\mathcal{I}\left(\tau ; P_{i}, I_{j}, \tau^{n+1}, \zeta_{i, j}(\tau)\right)$ denote a path satisfying (3.5), which arrives at a discrete grid point $\left(P_{i}, I_{j}\right)$ at $\tau=\tau^{n+1}$ for $P_{i}$ being held constant and control following a path $\zeta_{i, j}(\tau)$ with respect to $\tau$. Let $\mathcal{I}\left(\tau^{n} ; P_{i}, I_{j}, \tau^{n+1}, \zeta_{i, j}\left(\tau^{n}\right)\right)$ be the departure point of this path at $\tau=\tau^{n}$, which can be computed by solving

$$
\begin{cases}\frac{d \mathcal{I}}{d \tau}\left(\tau ; P_{i}, I_{j}, \tau^{n+1}, \zeta_{i, j}(\tau)\right)=\zeta_{i, j}(\tau)+a\left(\zeta_{i, j}(\tau)\right) & \text { for } \tau<\tau^{n+1}, \\ \mathcal{I}\left(\tau ; P_{i}, I_{j}, \tau^{n+1}, \zeta_{i, j}(\tau)\right)=I_{j} & \text { for } \tau=\tau^{n+1}\end{cases}
$$


from $\tau=\tau^{n+1}$ to $\tau=\tau^{n}$. We can write the solution of (3.8) in the integral form as

$$
\mathcal{I}\left(\tau=\tau^{n} ; P_{i}, I_{j}, \tau^{n+1}, \zeta_{i, j}\left(\tau=\tau^{n}\right)\right)=I_{j}-\int_{\tau^{n}}^{\tau^{n+1}}\left[\zeta_{i, j}(\tau)+a\left(\zeta_{i, j}(\tau)\right)\right] d \tau .
$$

Note that from (3.9), the departure point $\mathcal{I}\left(\tau^{n} ; P_{i}, I_{j}, \tau^{n+1}, \zeta_{i, j}\left(\tau^{n}\right)\right)$ will not necessarily coincide with a grid point in the $I$ direction. To simplify the notation, in the following we will use $\mathcal{I}(\tau)=$ $\mathcal{I}\left(\tau ; P_{i}, I_{j}, \tau^{n+1}, \zeta_{i, j}(\tau)\right)$ without causing ambiguity.

Integrating both sides of equation (3.7) along the path $\mathcal{I}(\tau)$ from $\tau=\tau^{n}$ to $\tau=\tau^{n+1}$, with $P$ fixed at $P_{i}$ and control variable $c$ following the path $\zeta_{i, j}(\tau)$, gives

$$
\begin{aligned}
& \int_{\tau^{n}}^{\tau^{n+1}} \min _{\zeta_{i, j}(\tau) \in C(\mathcal{I}(\tau))}\left\{\frac{D V}{D \tau}\left(P_{i}, \mathcal{I}(\tau), \tau\right)-\left(\zeta_{i, j}(\tau)-a\left(\zeta_{i, j}(\tau)\right)\right) P_{i}-\mathcal{L} V\left(P_{i}, \mathcal{I}(\tau), \tau\right)\right\} d \tau \\
& =0 .
\end{aligned}
$$

Interchanging the integral and the min operator in (3.10), assuming that they are interchangeable, and using the identity

$$
\int_{\tau^{n}}^{\tau^{n+1}} \frac{D V}{D \tau}\left(P_{i}, \mathcal{I}(\tau), \tau\right) d \tau=V\left(P_{i}, I_{j}, \tau^{n+1}\right)-V\left(P_{i}, \mathcal{I}\left(\tau^{n}\right), \tau^{n}\right)
$$

we obtain

$$
\begin{aligned}
V\left(P_{i}, I_{j}, \tau^{n+1}\right)= & \max _{\zeta_{i, j}(\tau) \in C(\mathcal{I}(\tau))}\left\{V\left(P_{i}, \mathcal{I}\left(\tau^{n}\right), \tau^{n}\right)+\int_{\tau^{n}}^{\tau^{n+1}}\left(\zeta_{i, j}(\tau)-a\left(\zeta_{i, j}(\tau)\right)\right) P_{i} d \tau\right. \\
& \left.+\int_{\tau^{n}}^{\tau^{n+1}} \mathcal{L} V\left(P_{i}, \mathcal{I}(\tau), \tau\right) d \tau\right\}
\end{aligned}
$$

where $\mathcal{I}(\tau)=\mathcal{I}\left(\tau ; P_{i}, I_{j}, \tau^{n+1}, \zeta_{i, j}(\tau)\right)$.

Remark 3.1 (Interchanging the order of operations in (3.10)). The integral and the min operator may not be interchangeable. Moreover, the derivatives in equation (3.10) may not exist since the value function $V$ may not be smooth and needs to be considered in the sense of the viscosity solution. Thus, our derivation is not rigorous. However, our purpose here is to illustrate the main idea for developing the schemes. The rigorous proof of the convergence of the semi-Lagrangian fully implicit discretization to the viscosity solution of equation (2.14) will be given in Section 4.

By evaluating the integrals in equation (3.9), the departure point of the semi-Lagrangian trajectory, and value function (3.12) using various numerical integration schemes, we are able to obtain semi-Lagrangian discretizations of different orders in time. In this paper, we will present the fully implicit and Crank-Nicolson timestepping schemes, which result from approximating the integrals using the rectangular rule and trapezoidal rule, respectively. We will use an approach similar to that suggested in [19]. 


\subsection{Fully implicit timestepping}

In the case of fully implicit timestepping, we approximate the integral in equation (3.9) using the rectangular rule at $\tau=\tau^{n+1}$. In other words, we evaluate (3.9) by assuming that

$$
\zeta_{i, j}(\tau) \simeq \zeta_{i, j}\left(\tau^{n+1}\right) \quad \text { for } \tau \in\left[\tau^{n}, \tau^{n+1}\right] .
$$

It is perhaps not immediately obvious why we evaluate $\zeta_{i, j}(\tau)$ at $\tau^{n+1}$ in approximation (3.13). In Appendix B we show that this choice corresponds to a discretization based on assuming that the operator of the facility can adjust the controls only at finite intervals, and that no-arbitrage jump conditions are applied at the control choice times. As a consequence, the fully implicit semi-Lagrangian discretization satisfies discrete no-arbitrage jump conditions.

Let $\zeta_{i, j}^{n+1}=\zeta_{i, j}\left(\tau^{n+1}\right)$ and let $\mathcal{I}_{j(i, n+1)}^{n}$ denote an approximation to $\mathcal{I}\left(\tau^{n}\right)=\mathcal{I}\left(\tau^{n} ; P_{i}, I_{j}, \tau^{n+1}, \zeta_{i, j}\left(\tau^{n}\right)\right)$, the departure point of the semi-Lagrangian trajectory (3.8). The rectangular approximation of (3.9), assuming (3.13), gives

$$
\mathcal{I}_{j(i, n+1)}^{n}=I_{j}-\Delta \tau^{n}\left(\zeta_{i, j}^{n+1}+a\left(\zeta_{i, j}^{n+1}\right)\right)
$$

where $\Delta \tau^{n}=\tau^{n+1}-\tau^{n}$.

The control $\zeta_{i, j}^{n+1}$ must satisfy the constraint $\zeta_{i, j}^{n+1} \in C\left(I_{j}\right)$, where $C\left(I_{j}\right)=\left[c_{\min }\left(I_{j}\right),-k_{5}\right] \cup$ $\left[0, c_{\max }\left(I_{j}\right)\right]$, as defined in equation (2.7). Moreover, to prevent the value of $\mathcal{I}_{j(i, n+1)}^{n}$ from going outside of the domain $\left[0, I_{\max }\right]$, we need to impose further constraints on $\zeta_{i, j}^{n+1}$. Let $C_{j}^{n+1} \subseteq C\left(I_{j}\right)$ denote the set of values of $\zeta_{i, j}^{n+1}$ such that the resulting $\mathcal{I}_{j(i, n+1)}^{n}$ calculated from equation (3.14) is bounded within $\left[0, I_{\max }\right]$. Note that $C_{j}^{n+1}$ is independent of $P_{i}$. We regard all elements in $C_{j}^{n+1}$ as admissible controls.

Equation (3.14) provides $\mathcal{I}_{j(i, n+1)}^{n}$ as an approximation to $\mathcal{I}\left(\tau^{n}\right)$. Hence, $V\left(P_{i}, \mathcal{I}_{j(i, n+1)}^{n}, \tau^{n}\right)$ is an approximation to $V\left(P_{i}, \mathcal{I}\left(\tau^{n}\right), \tau^{n}\right)$, which is the value function at $\tau^{n}$ when $P$ is fixed at $P_{i}$ and $I$ residing at the departure point of the path $\mathcal{I}(\tau)$. As mentioned above, $\mathcal{I}_{j(i, n+1)}^{n}$ usually does not coincide with a grid point in $I$ direction. Thus, we have to choose an interpolation scheme to approximate $V\left(P_{i}, \mathcal{I}_{j(i, n+1)}^{n}, \tau^{n}\right)$ using discrete grid values $V_{i, j}^{n}, i=1, \ldots, i_{\max }, j=1, \ldots, j_{\max }$. Let $V_{i, j(i, n+1)}^{n}$ denote an approximation of $V\left(P_{i}, \mathcal{I}_{j(i, n+1)}^{n}, \tau^{n}\right)$ obtained by interpolating a set of values $V_{i, j}^{n}$ with $P$ fixed at $P_{i}$ and $I$ varied.

Evaluating the integrals in (3.12) at $\tau=\tau^{n+1}$ using the rectangular rule, assuming that the control path $\zeta_{i, j}(\tau)$ follows (3.13) and the semi-Lagrangian trajectory $\mathcal{I}(\tau)$ satisfies $(3.14)$, gives

$$
V_{i, j}^{n+1}=\max _{\zeta_{i, j}^{n+1} \in C_{j}^{n+1}}\left\{V_{i, j(i, n+1)}^{n}+\Delta \tau^{n}\left(\zeta_{i, j}^{n+1}-a\left(\zeta_{i, j}^{n+1}\right)\right) P_{i}\right\}+\Delta \tau^{n}\left(\mathcal{L}_{h} V\right)_{i, j}^{n+1}
$$

where $V\left(P_{i}, \mathcal{I}\left(\tau^{n}\right), \tau^{n}\right)$ in (3.12) is approximated by $V_{i, j(i, n+1)}^{n}$. The last term in (3.15) follows from approximating the second integral in (3.12) assuming $\mathcal{L} V\left(P_{i}, \mathcal{I}(\tau), \tau\right)=\mathcal{L} V\left(P_{i}, \mathcal{I}\left(\tau^{n+1}\right), \tau^{n+1}\right)=$ $\mathcal{L} V\left(P_{i}, I_{j}, \tau^{n+1}\right)$ for $\tau \in\left[\tau^{n}, \tau^{n+1}\right]$ and then replacing the differential operator $\mathcal{L} V$ with its discrete form $\mathcal{L}_{h} V$, given in (3.3). Equations (3.14-3.15) specify a semi-Lagrangian fully implicit discretization. Assuming the solution value is smooth, although this is not the case in general, we show in Lemma 4.4 that linear interpolation for computing $V_{i, j(i, n+1)}^{n}$ is sufficient to achieve a first-order global discretization error. We will also demonstrate the first-order convergence of the fully implicit timestepping scheme using numerical experiments in Section 5. 
Using an entirely different approach, in Appendix B, we derive a semi-discretization based on a discrete optimal control approximation, and no-arbitrage jump conditions. If we further discretize this method in the $(P, \mathcal{I})$ directions, we obtain a discretization which is algebraically identical to the fully implicit discretization (3.14-3.15).

\subsection{Crank-Nicolson timestepping}

In order to obtain a higher order discretization in time, we can evaluate the integrals in (3.9) and (3.12) using a trapezoidal rule, which results in a Crank-Nicolson timestepping scheme. We assume that the control path $\zeta_{i, j}(\tau)$ is a continuous differentiable function of time. Let $\zeta_{i, j}^{n}=\zeta_{i, j}\left(\tau^{n}\right)$. Applying the trapezoidal rule to the integral in (3.9), assuming the control is a smooth function of time, gives the following approximation for $\mathcal{I}_{j(i, n+1)}^{n}$

$$
\mathcal{I}_{j(i, n+1)}^{n}=I_{j}-\frac{\Delta \tau^{n}}{2}\left(\zeta_{i, j}^{n+1}+a\left(\zeta_{i, j}^{n+1}\right)\right)-\frac{\Delta \tau^{n}}{2}\left(\zeta_{i, j}^{n}+a\left(\zeta_{i, j}^{n}\right)\right)
$$

Similar to the definition of admissible controls in the previous subsection, we can define $C_{j}^{n+1} \subseteq$ $C\left(\mathcal{I}_{j(i, n+1)}^{n}\right) \times C\left(I_{j}\right)$ be the set of all admissible controls $\zeta_{i, j}^{n}$ and $\zeta_{i, j}^{n+1}$ such that the value $\mathcal{I}_{j(i, n+1)}^{n}$ calculated from (3.16) resides inside the domain $\left[0, I_{\max }\right]$.

Approximating the integrals in (3.12) using the trapezoidal rule, assuming that the control path $\zeta_{i, j}(\tau)$ is a smooth function of time, and that the semi-Lagrangian trajectory $\mathcal{I}(\tau)$ follows (3.16), then we obtain

$$
\begin{aligned}
V_{i, j}^{n+1}= & \max _{\left(\zeta_{i, j}^{n}, \zeta_{i, j}^{n+1}\right) \in C_{j}^{n+1}}\left\{V_{i, j(i, n+1)}^{n}+\frac{\Delta \tau^{n}}{2}\left(\zeta_{i, j}^{n+1}-a\left(\zeta_{i, j}^{n+1}\right)\right) P_{i}\right. \\
& \left.+\frac{\Delta \tau^{n}}{2}\left(\zeta_{i, j}^{n}-a\left(\zeta_{i, j}^{n}\right)\right) P_{i}+\frac{\Delta \tau^{n}}{2}\left(\mathcal{L}_{h} V\right)_{i, j(i, n+1)}^{n}\right\}+\frac{\Delta \tau^{n}}{2}\left(\mathcal{L}_{h} V\right)_{i, j}^{n+1},
\end{aligned}
$$

where $\left(\mathcal{L}_{h} V\right)_{i, j(i, n+1)}^{n}$ is the evaluation of the discrete differential operator (3.3) at $\tau=\tau^{n}$ and $(P, I)=\left(P_{i}, \mathcal{I}_{j(i, n+1)}^{n}\right)$ with $\mathcal{I}_{j(n+1)}^{n}$ given in equation (3.16). Equations (3.16-3.17) results in a semi-Lagrangian Crank-Nicolson discretization.

As in the fully implicit timestepping case, we need to use interpolation to compute quantities of the form $(\cdot)_{i, j(i, n+1)}^{n}$ in $(3.17)$ since $\mathcal{I}_{j(n+1)}^{n}$ usually does not reside on a grid point in $I$ direction. As discussed in Remark 4.5, and as suggested by [15, 9, 20] for the case when the control is a fixed constant, second order global truncation error can be achieved if the $P$ derivatives in $\mathcal{L} V$ are discretized using second-order accurate methods, e.g., central differencing method (see Appendix A), and quadratic interpolation is used for $(\cdot)_{i, j(i, n+1)}^{n}$. Of course, this truncation error estimate is valid only for smooth solutions. Indeed, in the numerical experiments conducted in Section 5, we cannot observe second-order convergence for the Crank-Nicolson timestepping scheme with high-order interpolants. 


\subsection{Matrix form of the discrete equations}

We can write schemes (3.14-3.15) and (3.16-3.17) together using a general $\theta$-form as:

$$
\begin{aligned}
\mathcal{I}_{j(i, n+1)}^{n}= & I_{j}-(1-\theta) \Delta \tau^{n}\left(\zeta_{i, j}^{n+1}+a\left(\zeta_{i, j}^{n+1}\right)\right)-\theta \Delta \tau^{n}\left(\zeta_{i, j}^{n}+a\left(\zeta_{i, j}^{n}\right)\right), \\
& \mathcal{I}_{j(i, n+1)}^{n} \in\left[0, I_{\max }\right] \text { for any }\left(\zeta_{i, j}^{n}, \zeta_{i, j}^{n+1}\right) \in C_{j}^{n+1} ; \\
V_{i, j}^{n+1}= & \max _{\left(\zeta_{i, j}^{n}, \zeta_{i, j}^{n+1}\right) \in C_{j}^{n+1}}\left\{V_{i, j(i, n+1)}^{n}+(1-\theta) \Delta \tau^{n}\left(\zeta_{i, j}^{n+1}-a\left(\zeta_{i, j}^{n+1}\right)\right) P_{i}\right. \\
& \left.+\theta \Delta \tau^{n}\left(\zeta_{i, j}^{n}-a\left(\zeta_{i, j}^{n}\right)\right) P_{i}+\theta \Delta \tau^{n}\left(\mathcal{L}_{h} V\right)_{i, j(i, n+1)}^{n}\right\}+(1-\theta) \Delta \tau^{n}\left(\mathcal{L}_{h} V\right)_{i, j}^{n+1},
\end{aligned}
$$

where $\theta=0$ and $\theta=1 / 2$ correspond to the semi-Lagrangian fully implicit discretization and Crank-Nicolson discretization, respectively.

Before proceeding to setting up the matrix form for the discrete equations (3.18), let us introduce the following notation. Let $V_{j}^{n}$ be a column vector of discrete solution values with $\left[V_{j}^{n}\right]_{i}=V_{i, j}^{n}$ for a fixed $I_{j}$. Let $V^{n}$ be a column vector with $\left[V^{n}\right]_{j}=V_{j}^{n}$. Based on the discrete differential operator $\mathcal{L}_{h} V$ in (3.3), we can define a matrix $L$ such that

$$
\begin{aligned}
{\left[L \cdot V^{n}\right]_{i j} } & =\left(\mathcal{L}_{h} V\right)_{i, j}^{n} \\
& =\left[\alpha_{i}^{n} V_{i-1, j}^{n}+\beta_{i}^{n} V_{i+1, j}^{n}-\left(\alpha_{i}^{n}+\beta_{i}^{n}+r\right) V_{i, j}^{n}\right],
\end{aligned}
$$

where the coefficients $\alpha_{i}^{n}$ and $\beta_{i}^{n}$ are given in Appendix A.

Let $\Phi^{n+1}$ be a Lagrange interpolation operator such that

$$
\left[\Phi^{n+1} \cdot V^{n}\right]_{i, j}=V_{i, j(i, n+1)}^{n}+\text { interpolation error }
$$

where $\Phi^{n+1}$ can represent any order (linear, quadratic) of Lagrangian interpolation. Let $\left[\Phi^{n+1} V^{n}\right]_{j}$ denote a column vector with entries

$$
\left[\left[\Phi^{n+1} V^{n}\right]_{j}\right]_{i}=\left[\Phi^{n+1} V^{n}\right]_{i, j}
$$

Let $P$ denote a column vector satisfying $[P]_{i}=P_{i}$. Let $\zeta_{j}^{n}$ and $\zeta_{j}^{n+1}$ be diagonal matrices with diagonal entries $\left[\zeta_{j}^{n}\right]_{i i}=\zeta_{i, j}^{n}$ and $\left[\zeta_{j}^{n+1}\right]_{i i}=\zeta_{i, j}^{n+1}$. Similarly, let $a\left(\zeta_{j}^{n}\right)$ and $a\left(\zeta_{j}^{n+1}\right)$ denote diagonal matrices with diagonal entries $\left[a\left(\zeta_{j}^{n}\right)\right]_{i i}=a\left(\zeta_{i, j}^{n}\right),\left[a\left(\zeta_{j}^{n+1}\right)\right]_{i i}=a\left(\zeta_{i, j}^{n+1}\right)$. Let $I$ be an identity matrix. Given the above notations, the discrete equations (3.18) can be written as

$$
\begin{aligned}
& {\left[I-(1-\theta) \Delta \tau^{n} L V^{n+1}\right]_{j}=} {\left[\Phi^{n+1}\left[I+\theta \Delta \tau^{n} L\right] V^{n}\right]_{j}+} \\
&(1-\theta) \Delta \tau^{n}\left(\zeta_{j}^{n+1}-a\left(\zeta_{j}^{n+1}\right)\right) P+\theta \Delta \tau^{n}\left(\zeta_{j}^{n}-a\left(\zeta_{j}^{n}\right)\right) P \\
& \text { where }\left[\zeta_{j}^{n}\right]_{i},\left[\zeta_{j}^{n+1}\right]_{i}=\underset{\substack{\left.\left[\zeta_{j}^{n}\right]_{i},\left[\zeta_{j}^{n+1}\right]_{i}\right) \in C_{j}^{n+1} \\
\operatorname{argmax}}}{ }\left\{\left[\left[\Phi^{n+1}\left[I+\theta \Delta \tau^{n} L\right] V^{n}\right]_{j}+\right.\right. \\
&\left.\left.(1-\theta) \Delta \tau^{n}\left(\zeta_{j}^{n+1}-a\left(\zeta_{j}^{n+1}\right)\right) P+\theta \Delta \tau^{n}\left(\zeta_{j}^{n}-a\left(\zeta_{j}^{n}\right)\right) P\right]_{i}\right\}
\end{aligned}
$$

for $j=1, \ldots, j_{\max }$. Here $\theta=0$ corresponds to fully implicit timestepping, and $\theta=1 / 2$ is CrankNicolson timestepping. 
It will be convenient to define the following

$$
\begin{array}{cc}
\Delta P_{\max }=\max _{i}\left(P_{i+1}-P_{i}\right) ; \Delta P_{\min }=\min _{i}\left(P_{i+1}-P_{i}\right), \\
\Delta I_{\max }=\max _{j}\left(I_{j+1}-I_{j}\right) ; \Delta I_{\min }=\min _{j}\left(I_{j+1}-I_{j}\right), \\
\Delta \tau_{\max }=\max _{n}\left(\tau_{n+1}-\tau_{n}\right) ; \Delta \tau_{\min }=\min _{n}\left(\tau_{n+1}-\tau_{n}\right) .
\end{array}
$$

We assume that there are mesh size/timestep parameters $h_{\min }=C_{0} h_{\max }$ such that

$$
\begin{aligned}
& \Delta P_{\max }=C_{1} h_{\max } ; \Delta I_{\max }=C_{2} h_{\max } ; \Delta \tau_{\max }=C_{3} h_{\max } ; \\
& \Delta P_{\min }=C_{1}^{\prime} h_{\min } ; \Delta I_{\min }=C_{2}^{\prime} h_{\min } ; \Delta \tau_{\min }=C_{3}^{\prime} h_{\min } ;
\end{aligned}
$$

where $C_{0}, C_{1}, C_{1}^{\prime}, C_{2}, C_{2}^{\prime}, C_{3}, C_{3}^{\prime}$ are constants independent of $h_{\min }, h_{\max }$. We can write the discrete equations $(3.18)$ or $(3.22)$ at each node $\left(P_{i}, I_{j}\right)$ as

$$
\begin{aligned}
& G_{i, j}^{n+1}\left(h_{\max }, V_{i, j}^{n+1},\left\{V_{k, j}^{n+1}\right\}_{k \neq i},\left\{V_{i, j}^{n}\right\}\right) \\
& \equiv \min _{\left(\zeta_{i, j}^{n,}, \zeta_{i, j}^{n+1}\right) \in C_{j}^{n+1}}\left\{\frac{V_{i, j}^{n+1}-\left[\Phi^{n+1} V^{n}\right]_{i, j}}{\Delta \tau^{n}}-(1-\theta)\left[L V^{n+1}\right]_{i j}-\theta\left[\Phi^{n+1} L V^{n}\right]_{i, j}\right. \\
& \left.\quad-(1-\theta)\left[\left(\zeta_{j}^{n+1}-a\left(\zeta_{j}^{n+1}\right)\right) P\right]_{i}-\theta\left[\left(\zeta_{j}^{n}-a\left(\zeta_{j}^{n}\right)\right) P\right]_{i}\right\} \\
& =0,
\end{aligned}
$$

where $\left\{V_{k, j}^{n+1}\right\}_{k \neq i}$ is the set of values $V_{k, j}^{n+1}, k \neq i, k=1, \ldots, i_{\max }$, and $\left\{V_{i, j}^{n}\right\}$ is the set of values $V_{i, j}^{n}, i=1, \ldots, i_{\max }, j=1, \ldots, j_{\max }$.

\section{Convergence to the Viscosity Solution}

The pricing PDE (2.14) is nonlinear, hence the solution to the PDE may not be unique [13, 2]. As pointed out in $[22,2]$, it is important to ensure that a numerical scheme for solving PDE (2.14) converges to the valid solution from a financial perspective, which in this case is the viscosity solution. Provided a strong comparison result for the PDE applies, [7, 2] demonstrate that a numerical scheme will converge to the viscosity solution of the equation if it is $l_{\infty}$ stable, pointwise consistent, and monotone. Schemes failing to satisfy these conditions may converge to non-viscosity solutions. In fact, [30] gives an example where seemingly reasonable discretizations of nonlinear option pricing PDEs that do not satisfy the sufficient convergence conditions for viscosity solutions either never converge or converge to a non-viscosity solution.

We first remark on the existence of the strong comparison result for equation (2.14). 


\subsection{Strong comparison result}

The PDE together with the terminal conditions and boundary conditions in Section 2.4 can be written as

$$
\begin{array}{r}
V_{\tau}-\frac{1}{2} \sigma^{2} P^{2} V_{P P}-\mu(P, t) V_{P}-\max _{c \in C(I)}\left\{(c-a(c)) P-(c+a(c)) V_{I}\right\}+r V=0 \\
\text { when }(P, I, \tau) \in\left(0, P_{\max }\right) \times\left[0, I_{\max }\right] \times(0, T) ; \\
V_{\tau}-\mu(P, t) V_{P}-\max _{c \in C(I)}\left\{(c-a(c)) P-(c+a(c)) V_{I}\right\}+r V=0 \\
\text { when }(P, I, \tau) \in\left\{0, P_{\max }\right\} \times\left[0, I_{\max }\right] \times(0, T) ; \\
V=V(P, I, 0) \quad ; \quad \text { when }(P, I, \tau) \in\left[0, P_{\max }\right] \times\left[0, I_{\max }\right] \times\{0\},
\end{array}
$$

where

$$
\begin{aligned}
c+a(c) & \leq 0 \text { as } I \rightarrow 0 ; \quad c+a(c) \geq 0 \text { as } I \rightarrow I_{\max } \\
\mu(P, t) & \geq 0 \text { as } P \rightarrow 0 ; \quad \mu(P, t) \leq 0 \text { as } P \rightarrow P_{\max }
\end{aligned}
$$

and $V(P, I, 0)$ is the payoff of the gas storage contract. Provided that the control variable is a constant, and we assume that $\sigma \rightarrow 0$ smoothly as $P \rightarrow P_{\max }$, the results in [26] indicate that there exists a classical solution for equation (2.14) since the characteristics in $P$ and $I$ directions are outgoing at the boundary. Note that we also require that the diffusion term $\sigma$ goes to zero as $P \rightarrow 0, P_{\max }$ at a sufficiently high rate for this result to hold. If the classical solution exists, the strong comparison result holds in the constant control case according to the definition given in $[7,2]$.

With the appearance of the optimal control, the equation turns into an HJB equation. There are various research papers deriving a strong comparison result for second-order HJB equations associated with several types of boundary conditions $[4,6,12,3,10]$. In particular, $[4,6]$ prove that the viscosity solution of degenerate elliptic HJB equations with Dirichlet boundary conditions satisfies the strong comparison result, provided that several assumptions are satisfied. In [6], the author demonstrates that

$\mathbf{S 1}$ if the coefficient of the diffusion term (in our case $\sigma^{2} P^{2}$ ) vanishes at a region on a boundary with an outgoing characteristic, independent of the value for the control variable, then the viscosity solution on this boundary region is the limit of the viscosity solution from interior points;

S2 if the characteristic at a region on the boundary, associated with the first order term in the $\mathrm{PDE}$, is incoming to the domain independent of the choice of the control value, then the viscosity solution at the region corresponds to the specified boundary data in the classical sense.

We can regard the two-dimensional parabolic PDE (2.14) as a three-dimensional degenerateelliptic PDE in the variable $x=(\tau, P, I) \in \mathbb{R}^{N} \times \mathbb{R}^{N} \times\left[0, I_{\max }\right]$. The resulting elliptic PDE is degenerate in the sense that the equation does not contain the second-order derivatives with respect to $\tau$ and $I$, or, equivalently, the effective volatility (i.e. the diffusion term) is zero with respect to $\tau$ and $I$. From equations (4.1) and (4.4), the statement $\mathbf{S} 1$ above is satisfied for the boundary region $(P, I, \tau) \in\left[0, P_{\max }\right] \times\left\{0, I_{\max }\right\} \times(0, T)$. Similarly, statement $\mathbf{S} 1$ above is also 
satisfied when $(P, I, \tau) \in\left\{0, P_{\max }\right\} \times\left[0, I_{\max }\right] \times(0, T)$ according to equations (4.2) and (4.4). Thus, the viscosity solution does not require boundary data in both $P$ and $I$ directions, which confirms our intuition in setting the boundary conditions in these directions. Equation (4.1) implies that statement $\mathbf{S} 2$ above is satisfied when $(P, I, \tau) \in\left[0, P_{\max }\right] \times\left[0, I_{\max }\right] \times\{0\}$. This means that the viscosity solution uses the Dirichlet boundary condition, which we provided as the payoff function in equation (4.3).

From the analysis above, the boundary conditions we apply for equation (2.14) are in accordance with the behavior of the viscosity solution at the boundary. Consequently, we can use the strong comparison result in [4, 6] if equations (4.1-4.4) satisfy assumptions given in [4, 6]. However, technical difficulties arise when we try to verify two assumptions among those outlined in $[4,6]$. First, the boundary is assumed to be smooth in $[4,6]$ so that the normal vectors at the boundary are all well-defined. In our case, however, the boundary surface is a cuboid, which results in the non-smoothness in the corners of the cuboid. Second, in $[4,6]$, the coefficient of the diffusion term is required to be continuous in the domain. In our case, the volatility $\sigma(P)$ is constant inside the domain and zero at $P=P_{\max }$, which causes a discontinuity at the boundary. However, we can overcome these difficulties by defining appropriate normal vectors at the non-smooth corners and using a smooth mollifier to approximate the discontinuity of the volatility on the boundary. To avoid tedious complication, we omit the detailed construction required to eliminate this technical difficulty and assume that equations (4.1-4.4) satisfy the strong comparison result.

We will show that the semi-Lagrangian fully implicit discretization (equation (3.18), (3.22), or $(3.25)$, with $\theta=0)$ converges to the viscosity solution of equation (2.14) by verifying the stability, consistency, and monotonicity of the scheme in sequence. As noted in Remark 4.5, higher than or equal to third-order (quadratic) interpolation is needed for operation $\Phi^{n+1}$ in (3.25) in order to achieve a second-order global truncation error for Crank-Nicolson timestepping (for smooth solutions). This makes this scheme non-monotone in general, and hence we cannot guarantee convergence of high-order Crank-Nicolson timestepping to the viscosity solution because monotonicity can be obtained only for linear interpolation. We will, nevertheless, prove the consistency of the Crank-Nicolson timestepping scheme and carry out numerical experiments with Crank-Nicolson timestepping using quadratic interpolation.

\subsection{Stability}

In this section, we demonstrate the $l_{\infty}$ stability of the scheme (3.18).

Definition 4.1 (Stability). Discretization (3.18) is $l_{\infty}$ stable if

$$
\left\|V^{n+1}\right\|_{\infty} \leq C_{4}
$$

for $0 \leq n \leq N$ as $\Delta \tau_{\min } \rightarrow 0, \Delta P_{\min } \rightarrow 0, \Delta I_{\min } \rightarrow 0$, where $C_{4}$ is a constant independent of $\Delta \tau_{\min }, \Delta P_{\min }, \Delta I_{\min }$. Here $\left\|V^{n+1}\right\|_{\infty}=\max _{i, j}\left|V_{i, j}^{n+1}\right|$.

The stability of the semi-Lagrangian fully implicit discretization (3.18) is a consequence of the following Lemma.

Lemma 4.2 (Stability of the fully implicit scheme). Assuming that discretization (3.3) satisfies the positive coefficient condition (3.4) and linear interpolation is used to compute $V_{i, j(i, n+1)}^{n}$, then the scheme (3.18) satisfies

$$
\left\|V^{n+1}\right\|_{\infty} \leq\left\|V^{0}\right\|_{\infty}+C_{5}
$$


in the case of fully implicit timestepping $(\theta=0)$, where $C_{5}=T \cdot P_{\max } \cdot \max \left\{\left|c_{\max }\left(I_{\max }\right)\right|,\left|c_{\min }(0)\right|\right\}$.

Proof. The proof directly follows from applying the maximum principle to the discrete equation (3.18). We omit the details here. Readers can refer to [15, Theorem 5.5] and [22] for complete stability proofs of the semi-Lagrangian fully implicit scheme for American Asian options and that of finite difference schemes for controlled HJB equations, respectively.

\subsection{Consistency}

We give in the following a definition for the consistency of a discretization.

Definition 4.3 (Consistency). The discretization $G_{i, j}^{n+1}\left(h_{\max }, V_{i, j}^{n+1},\left\{V_{k, j}^{n+1}\right\}_{k \neq i},\left\{V_{i, j}^{n}\right\}\right)$ given in equation (3.25) is consistent if, for any smooth test function $\phi(S, P, \tau)$ having bounded derivatives of all orders, with $\phi_{i, j}^{n}=\phi\left(S_{i}, P_{j}, \tau^{n}\right)$, we have that:

$$
\begin{gathered}
\lim _{h_{\max } \rightarrow 0} \mid \min _{c \in C\left(I_{j}\right)}\left\{\left(\phi_{\tau}+(c+a(c)) \phi_{I}-(c-a(c)) P-\mathcal{L} \phi\right)_{i, j}^{n+1}\right\}- \\
G_{i, j}^{n+1}\left(h_{\max }, \phi_{i, j}^{n+1},\left\{\phi_{k, j}^{n+1}\right\}_{k \neq i},\left\{\phi_{i, j}^{n}\right\}\right) \mid=0 .
\end{gathered}
$$

Note that in order to handle the boundary data, the consistency condition is more complicated if general Dirichlet or Neumann boundary conditions are specified [7, 2]. In our case, the boundary conditions specified in Section 2.4 are the limit of equation (2.14) as $P, I$ goes towards the boundary (see also equations (4.1-4.4)). As a result, the need for this more general condition does not occur.

Lemma 4.4 (Consistency). Suppose the mesh size and timestep size satisfy equations (3.24), and the control parameters satisfy condition (2.7). Then the discretization (3.25) is consistent as defined in Definition 4.3, provided that $c_{\max }, c_{\min }$ and $a(c)$ satisfy equations (2.1), (2.3) and (2.5), respectively.

Proof. See Appendix D.

Remark 4.5 (Crank-Nicolson truncation error). In ([19, Theorem 3.3]), a second-order truncation error is established for the Heun discretization of a time-independent first-order controlled HJB equation, assuming that the solution is smooth. Following the method in [19], if we assume that the solution is sufficiently smooth, central weighting is used for operator $\mathcal{L}_{h} V$, quadratic interpolation is applied for operation $\Phi^{n+1}$, and the optimal control c satisfying

$$
c=\underset{c \in C\left(I_{j}\right)}{\arg \min }\left\{(c+a(c)) V_{I}\left(P_{i}, I_{j}, \tau^{n+1 / 2}\right)-(c-a(c)) P_{i}\right\}
$$

is attainable by both controls $\zeta_{i, j}^{n}$ and $\zeta_{i, j}^{n+1}$ in discretization (3.25), where $\tau^{n+1 / 2}=\left(\tau^{n}+\tau^{n+1}\right) / 2$, then the discretization (3.25) in the case of Crank-Nicolson timestepping $(\theta=1 / 2)$ results in a second-order global truncation error $O\left(\left(h_{\max }\right)^{2}\right)$. 


\subsection{Monotonicity}

In this section, we discuss the monotonicity of the fully implicit scheme (3.18).

Definition 4.6 (Monotonicity). The discretization $G_{i, j}^{n+1}\left(h_{\max }, V_{i, j}^{n+1},\left\{V_{k, j}^{n+1}\right\}_{k \neq i},\left\{V_{i, j}^{n}\right\}\right)$ given in equation (3.25) is monotone if

$$
\begin{gathered}
G_{i, j}^{n+1}\left(h_{\max }, V_{i, j}^{n+1},\left\{V_{k, j}^{n+1}+\epsilon_{k, j}^{n+1}\right\}_{k \neq i},\left\{V_{i, j}^{n}+\epsilon_{i, j}^{n}\right\}\right) \leq G_{i, j}^{n+1}\left(h_{\max }, V_{i, j}^{n+1},\left\{V_{k, j}^{n+1}\right\}_{k \neq i},\left\{V_{i, j}^{n}\right\}\right) ; \\
\forall i, j, \forall \epsilon_{i, j}^{n}, \epsilon_{i, j}^{n+1} \geq 0,
\end{gathered}
$$

and, for any constants $m, m_{0} \geq 0$, we have

$$
\begin{array}{r}
G_{i, j}^{n+1}\left(h_{\max }, V_{i, j}^{n+1}+m+m_{0} \tau^{n+1},\left\{V_{k, j}^{n+1}+m+m_{0} \tau^{n+1}\right\}_{k \neq i},\left\{V_{i, j}^{n}+m+m_{0} \tau^{n}\right\}\right) \\
\geq m_{0}+G_{i, j}^{n+1}\left(h_{\max }, V_{i, j}^{n+1},\left\{V_{k, j}^{n+1}\right\}_{k \neq i},\left\{V_{i, j}^{n}\right\}\right) ; \forall i, j, \forall m, m_{0} \geq 0 .
\end{array}
$$

This definition of monotonicity is equivalent to that introduced in [24].

Remark 4.7 (Interpretation of the definition of monotonicity). It can be shown that condition (4.9) can be deduced from the condition (4.8) and the consistency of the scheme (3.25). Specifically, [22] show that for $m_{0}>0, m=0$, condition (4.9) is equivalent to requiring that the discrete equations contain a consistent approximation to the $V_{\tau}$ term in PDE (2.14); for $m_{0}=0, m>0$, as outlined in [15, Remark 5.1], condition (4.9) will be satisfied for any consistent discretization of equation (2.14) provided that $r>0$ and condition (4.8) is satisfied. As noted in [15, 22], the monotonicity conditions imply that $V_{i, j}^{n+1}$ is a non-decreasing function of $\left\{V_{k, j}^{n+1}\right\}_{k \neq i}$ and $\left\{V_{i, j}^{n}\right\}$, which amounts to a discrete arbitrage inequality (refer to [22] for more details).

Lemma 4.8 (Monotonicity). If the discretization (3.3) satisfies the positive coefficient condition (3.4) and linear interpolation is used to compute $V_{i, j(i, n+1)}^{n}$, then in the case of fully implicit timestepping $(\theta=0)$, the discretization $G_{i, j}^{n+1}\left(h_{\max }, V_{i, j}^{n+1},\left\{V_{k, j}^{n+1}\right\}_{k \neq i},\left\{V_{i, j}^{n}\right\}\right)$, as given in (3.25), is monotone according to Definition 4.6.

Proof. The proof directly follows that of monotonicity of finite difference schemes for controlled HJB equations in $[5,22]$. Note that the proof assumes the consistency of the discretization (3.25), which has been shown in Lemma 4.4.

From Lemmas 4.2, 4.4 and 4.8, using the results in [7, 2], we can obtain the following convergence result:

Theorem 4.9 (Convergence to the viscosity solution). Assume that discretization (3.22) satisfies all the conditions required for Lemmas 4.2, 4.4 and 4.8, and that PDE (4.1-4.3) satisfies the strong comparison result, then scheme (3.22) converges to the viscosity solution of equation (2.14) in the case of fully implicit timestepping $(\theta=0)$.

\subsection{Solution algorithm}

The solution to equation (2.14) can be computed using Algorithm 4.10. 
$V^{0}=$ Option Payoff

For $n=0, \ldots, \quad / /$ Timestep loop

For $j=1, \ldots, / /$ Loop through nodes in $I$ direction

For $i=1, \ldots, / /$ Loop through nodes in $P$ direction

$$
\begin{array}{r}
{\left[\zeta_{j}^{n}\right]_{i},\left[\zeta_{j}^{n+1}\right]_{i}=\underset{\left(\left[\zeta_{j}^{n}\right]_{i},\left[\zeta_{j}^{n+1}\right]_{i}\right) \in C_{j}^{n+1}}{\operatorname{argmax}}\left\{\left[\left[\Phi^{n+1}\left[I+\theta \Delta \tau^{n} L\right] V^{n}\right]_{j}+\right.\right.} \\
\left.\left.(1-\theta) \Delta \tau^{n}\left(\zeta_{j}^{n+1}-a\left(\zeta_{j}^{n+1}\right)\right) P+\theta \Delta \tau^{n}\left(\zeta_{j}^{n}-a\left(\zeta_{j}^{n}\right)\right) P\right]_{i}\right\}
\end{array}
$$

EndFor

Solve

$$
\begin{aligned}
& {\left[I-(1-\theta) \Delta \tau^{n} L V^{n+1}\right]_{j}=\left[\Phi^{n+1}\left[I+\theta \Delta \tau^{n} L\right] V^{n}\right]_{j}+} \\
& \quad(1-\theta) \Delta \tau^{n}\left(\zeta_{j}^{n+1}-a\left(\zeta_{j}^{n+1}\right)\right) P+\theta \Delta \tau^{n}\left(\zeta_{j}^{n}-a\left(\zeta_{j}^{n}\right)\right) P
\end{aligned}
$$

EndFor

EndFor

Remark 4.10. As described in [22], a standard finite difference discretization for equation (2.14) requires an iteration at each timestep to solve the nonlinear discretized algebraic equations. However, Algorithm 4.10, which uses a semi-Lagrangian discretization, avoids the need for such nonlinear iterations.

Remark 4.11. At each timestep in Algorithm 4.10 all discrete equations in the I direction are decoupled and independent of each other. This property makes solution of the gas storage contract straightforward to implement, given an existing library that supports pricing of path-dependent options.

Note that for a finite grid size, the solution of the discrete control problem in Algorithm 4.10 may allow controls which are not possible controls for the exact solution of the HJB equation. The exact solution of equation (2.14) has the property that the controls are of the bang-bang type [32], i.e. the optimal controls can take on only the values in a finite set. Consequently, there are two possible approaches for determining the optimal controls at each grid node. We can use our knowledge of the exact controls to search only for optimal controls within the known finite set of possible values. We refer to this approach as the bang-bang method. Alternatively, we can simply solve the discrete optimization problem in Algorithm 4.10, and allow any admissible control. We will refer to this technique as the no bang-bang method. Of course, both methods will converge to the correct solution, with bang-bang type controls, for sufficiently small mesh spacing and timestep size. In Appendix $\mathrm{C}$ we provide the details for solving the local optimization problems in Algorithm 4.10. 


\section{$5 \quad$ Numerical Experiments}

Having presented several semi-Lagrangian discretization schemes in the previous sections, in this section, we conduct numerical experiments based on these schemes. We use "dollars per million British thermal unit" (\$/mmBtu) and "million cubic feet" (MMcf) as the default units for gas spot price $P$ and gas inventory $I$, respectively. Since $1000 \mathrm{mmBtus}$ are roughly equal to $1 \mathrm{MMcf}$, in order to unify the units, we need to multiply gas spot price by 1000 when computing payoffs or revenues.

Throughout the numerical experiments, we use the following non-smooth payoff function from [11]

$$
V(P, I, t=T)=-2 P \max (1000-I, 0) .
$$

Equation 5.1 indicates that severe penalties are charged if the gas inventory is less than 1000 MMcf and no compensation is received when the inventory is above 1000 MMcf. Naturally, such a payoff structure will force the operator of a gas storage facility to maintain the gas inventory as close to $1000 \mathrm{MMcf}$ as possible at maturity to avoid revenue loss.

This section is arranged as follows: we first give numerical results for the case without incorporating the seasonality effect into the equilibrium natural gas spot price; we then incorporate the seasonality feature and illustrate its influence on both the solution value and the optimal control strategy. At the end of this section, we further extend the underlying risk adjusted gas spot price process to include a compound Poisson process that simulates random jumps of the gas prices, and then present numerical results incorporating a jump diffusion process.

\subsection{No seasonality effect}

In this subsection, we assume that the equilibrium gas price is independent of time, that is, we set $K(t)=K_{0}$ in equation (2.9).

We first carry out a convergence analysis assuming that the risk adjusted natural gas spot price follows the pure mean-reverting process (2.8) with $\alpha=2.38, K_{0}=6, \sigma=0.59$. In other words, the risk adjusted gas spot price follows

$$
d P=2.38(6-P) d t+0.59 P d Z \text {. }
$$

We are most interested in the solution when the gas spot price is near the long-term equilibrium price, i.e., $P=6 \$ / \mathrm{mmBtu}$ for (5.2). Note that when $I=1000 \mathrm{MMcf}$, the payoff is non-smooth (see equation 5.1). Consequently, to fully test our semi-Lagrangian discretization schemes, we focus on the convergence results at $(P, I)=(6,1000)$. We use an unequally spaced grid in the $P, I$ directions, where there are more nodes around the mesh point $(P, I)=(6,1000)$, compared with other locations.

Table 5.1 lists other input parameters for pricing the value of a gas storage contract. The convergence results obtained from refining the mesh spacing and timestep size are shown in Table 5.2, where we use fully implicit and Crank-Nicolson timestepping schemes associated with both the bang-bang and no bang-bang methods for solving the discrete optimization problem in Algorithm 4.10 (refer to Section C). Linear interpolation and quadratic interpolation are used for fully implicit and Crank-Nicolson timestepping, respectively. (Refer to Section 3 for a discussion on interpolation schemes.) Following [30], in order to improve the convergence for non-smooth payoff (5.1), we use a modification suggested by [31] for Crank-Nicolson timestepping. Specifically, we 


\begin{tabular}{cccc}
\hline Parameter & Value & Parameter & Value \\
\hline$r$ & 0.1 & $k_{2}$ & 730000 \\
$T$ & 3 years & $k_{3}$ & 500 \\
$I_{\max }$ & $2000 \mathrm{MMcf}$ & $k_{4}$ & 2500 \\
$k_{1}$ & 2040.41 & $k_{5}$ & $1.7 \cdot 365$ \\
\hline
\end{tabular}

TABLE 5.1: Input parameters used to price the value of a gas storage contract, where $I_{\max }$ is the maximum storage inventory; $k_{1}, k_{2}, k_{3}, k_{4}, k_{5}$ are parameters in equations (2.1-2.2) and (2.5). The values of $I_{\max }, k_{1}, k_{2}, k_{3}, k_{4}, k_{5}$ are taken from [32].

\begin{tabular}{ccc|cc|cc}
\hline \multirow{2}{*}{$\begin{array}{c}P \text { grid } \\
\text { nodes }\end{array}$} & $\begin{array}{c}\text { grid } \\
\text { nodes }\end{array}$ & No. of & \multicolumn{2}{c|}{ Bang-bang method } & \multicolumn{2}{c}{ No bang-bang method } \\
\cline { 4 - 7 } & timesteps & Value & Ratio & Value & Ratio \\
\hline \multicolumn{7}{c}{ Fully implicit timestepping } \\
\hline 53 & 61 & 500 & 4477036 & n.a. & 4556380 & n.a. \\
105 & 121 & 1000 & 4503705 & n.a. & 4542845 & n.a. \\
209 & 241 & 2000 & 4514723 & 2.42 & 4534660 & 1.63 \\
417 & 481 & 4000 & 4519809 & 2.17 & 4530331 & 1.89 \\
833 & 961 & 8000 & 4522653 & 1.79 & 4528219 & 2.05 \\
\hline \multicolumn{7}{c}{ Crank-Nicolson timestepping } \\
\hline 53 & 61 & 500 & 4483667 & n.a. & 4520475 & n.a. \\
105 & 121 & 1000 & 4509076 & n.a. & 4525352 & n.a. \\
209 & 241 & 2000 & 4517960 & 2.86 & 4526280 & 5.26 \\
417 & 481 & 4000 & 4522632 & 1.90 & 4527225 & 0.98 \\
833 & 961 & 8000 & 4524948 & 2.02 & 4527331 & 8.92 \\
\hline
\end{tabular}

TABle 5.2: Value of a natural gas storage facility at $P=6 \$ / \mathrm{mmBtu}$ and $I=1000 \mathrm{MMcf}$. Risk adjusted gas spot price follows the pure mean-reverting process (5.2). Convergence ratios are presented for fully implicit and Crank-Nicolson timestepping schemes with the bang-bang and the no bang-bang methods. Constant timesteps are used. Payoff function is given in (5.1). Other input parameters are given in Table 5.1. Crank-Nicolson incorporates the modification suggested in [31].

apply fully implicit timestepping in the first four timesteps, and use Crank-Nicolson timestepping in the rest of the timesteps.

The results in Table 5.2 indicate that both timestepping schemes converge to the same solution, although convergence to the viscosity solution can only be guaranteed for fully implicit timestepping. We define the convergence ratio as the ratio of successive changes in the solution, as the timestep and mesh size are reduced by a factor of two. A ratio of two indicates first order convergence, while a ratio of four indicates second order convergence. The convergence ratios are approximately two for fully implicit timestepping with both the bang-bang and the no bang-bang methods. Note that the no bang-bang method is a more general approach which can be used in cases where controls are not of bang-bang type.

It is interesting to note that in a fixed refinement level, the bang-bang method results in a smaller value than the no bang-bang method for both timestepping schemes. This is because the no bang-bang method actually solves the discrete optimization problem in Algorithm 4.10, instead 


\begin{tabular}{c|ccc|ccc}
\hline \multirow{2}{*}{ Timesteps } & \multicolumn{3}{|c|}{$P_{\max }=2000$} & \multicolumn{3}{c}{$P_{\max }=20000$} \\
\cline { 2 - 7 } & $P$ grid nodes & $I$ grid nodes & Value & $P$ grid nodes & $I$ grid nodes & Value \\
\hline 1600 & 53 & 61 & 4477036 & 57 & 61 & 4477036 \\
3200 & 105 & 121 & 4503705 & 113 & 121 & 4503705 \\
6400 & 209 & 241 & 4514723 & 225 & 241 & 4514723 \\
12800 & 417 & 481 & 4519809 & 449 & 481 & 4519809 \\
25600 & 833 & 961 & 4522653 & 897 & 961 & 4522653 \\
\hline
\end{tabular}

TABLE 5.3: Value of a natural gas storage facility at $P=6 \$ / m m B t u$ and $I=1000$ MMcf obtained using fully implicit timestepping with the bang-bang method. The right boundary of the domain $\left[0, P_{\max }\right]$ is truncated at different values of $P_{\max }$. Constant timesteps are used. Risk adjusted gas spot price follows the pure mean-reverting process (5.2). Payoff function is given in (5.1). Other input parameters are given in Table 5.1.

of only testing a finite set of points, which results in a higher solution value for PDE (2.14) (for a finite grid size) than the bang-bang method.

Table 5.2 also shows that Crank-Nicolson timestepping does not appear to converge at a secondorder rate. We have observed this same effect in many of our tests. Since we do not seem to obtain any benefit from Crank-Nicolson timestepping, fully implicit timestepping appears to be a better choice since we are guaranteed convergence to the viscosity solution, as shown in Section 4 . In the rest of this paper, we will use fully implicit timestepping exclusively.

Figure 5.1 shows the value surface of a gas storage contract at current time $t=0$ as a function of $P$ and $I$. The surface has a similar shape as that presented in [32]. The surface reaches a maximum value when the gas spot price is at the highest and the gas storage is full; the surface reaches the minimum point when the gas price is at the highest and the gas storage is empty. For a fixed $I$ around 1000 MMcf, the value surface also exhibits a parabolic shape in the $P$ direction.

Figure 5.2 shows the optimal control strategy that evolves over time when the inventory is fixed at $I=1000$ MMcf. From Figure 5.2, for a fixed time, the optimal control is a piece-wise constant function of $P$. The figure suggests that the optimal strategy is to inject gas at the maximum rate (corresponding to the flat negative control region in the surface) when the gas price is low, to produce gas at the maximum rate (corresponding to the positive control region) when the gas price is high, and to do nothing (corresponding to the zero control region) when the gas price is near the long-term equilibrium price $K_{0}=6 \$ / \mathrm{mmBtu}$. We can also observe that when the contract is close to maturity, the zero control region expands rapidly. This phenomenon is caused by the payoff function (5.1): at $I=1000 \mathrm{MMcf}$, when close to maturity, the operator tends to stop producing gas to avoid the severe penalty at maturity. In addition, the operator will stop injecting, since any leftover gas is lost to the operator.

Figure 5.3 shows the optimal control strategy surface at $t=0$ as a function of $P$ and $I$. This surface is similar to that given in [32]. The interpretation given in [32] also applies to Figure 5.3.

Our numerical computations truncate the domain $P \in[0, \infty]$ to $\left[0, P_{\max }\right]$. In order to test the influence of the domain truncation on the solution, in Table 5.3 we show the solution values at $P=6 \$ / \mathrm{mmBtu}, I=1000 \mathrm{MMcf}$ using two different values of $P_{\max }$. This table indicates that by setting $P_{\max }=2000 \$ / \mathrm{mmBtus}$, there is a negligible solution error incurred by the domain truncation. As a result, all subsequent results will be reported using $P_{\max }=2000 \$ / \mathrm{mmBtus}$.

In [32], a fully explicit timestepping method is used to solve the gas storage equation. The 


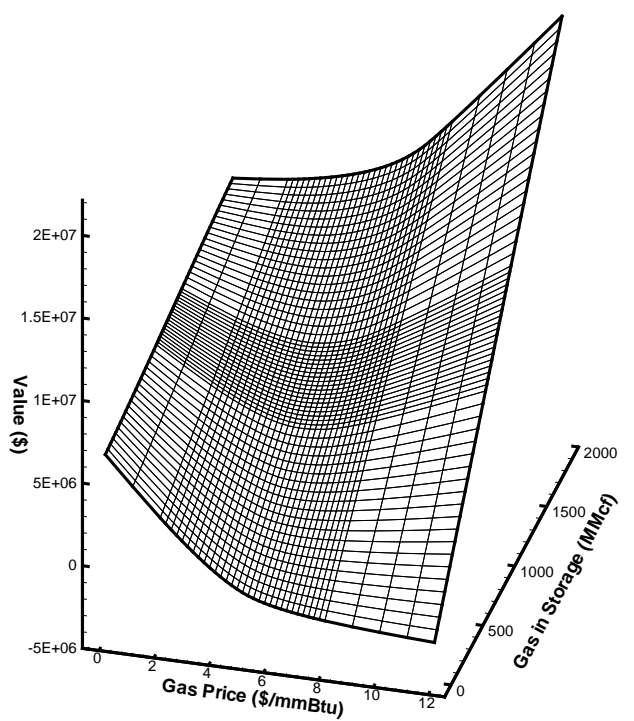

FigURE 5.1: Value surface of a gas storage contract at current time $t=0$ as a function of gas spot price $P$ and gas inventory I obtained using fully implicit timestepping with the no bang-bang method. Constant timesteps are used. Risk adjusted gas spot price follows the pure mean-reverting process (5.2). Payoff function is given in (5.1). Other input parameters are given in Table 5.1.

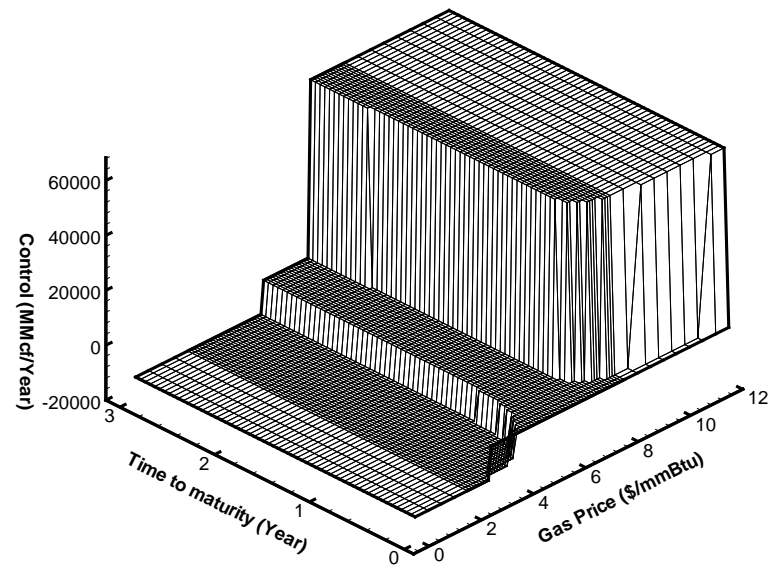

Figure 5.2: Optimal control strategy as a function of time-to-maturity $\tau=T-t$ and gas spot price $P$ when the gas inventory resides at $I=1000$ MMcf. Risk adjusted gas spot price follows the pure mean-reverting process (5.2). Payoff function is given in (5.1). Other input parameters are given in Table 5.1. Fully implicit timestepping with the no bang-bang method and with constant timesteps is used. 


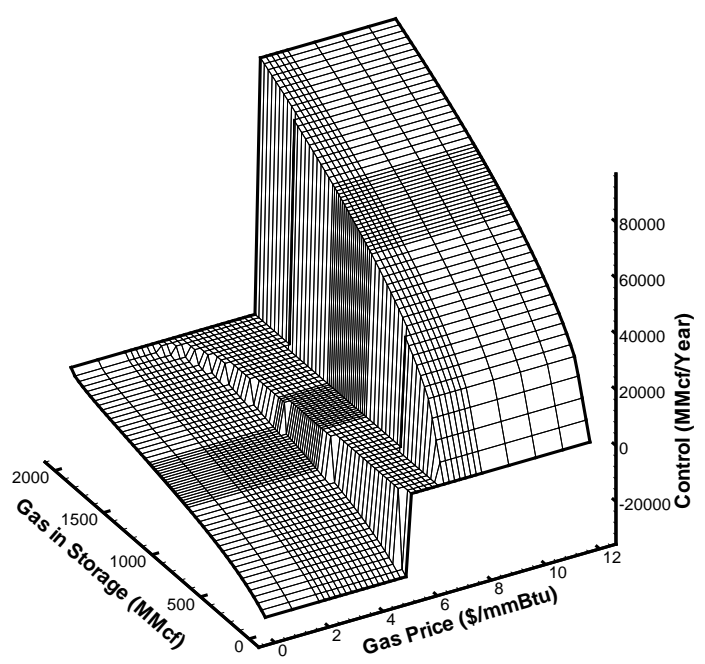

Figure 5.3: Optimal control strategy at current time $t=0$ as a function of gas spot price $P$ and gas inventory I. Risk adjusted gas spot price follows the pure mean-reverting process (5.2). Payoff function is given in (5.1). Other input parameters are given in Table 5.1. Fully implicit timestepping with the no bang-bang method and with constant timesteps is used.

explicit timestepping scheme requires the stability condition

$$
\Delta \tau^{n}<\min _{i}\left\{\frac{1}{\alpha_{i}^{n}+\beta_{i}^{n}+r}\right\},
$$

where parameters $\alpha_{i}^{n}, \beta_{i}^{n}$ are given in Appendix A. Condition (5.3) implies that $\Delta \tau^{n}=O\left((\Delta P)^{2}\right)$, for the case where $\Delta P=P_{i+1}-P_{i}=$ const.. In contrast, there is no such timestep restriction for fully implicit timestepping.

In Table 5.4, for different refinement levels, we compare the timestep size of implicit timestepping in our experiments with the maximum timestep size computed from condition (5.3) based on the same mesh discretization, which is approximately the maximum timestep allowed by the explicit timestepping. Table 5.4 shows that in the finest $(P, I)$ mesh, our timestep is more than fifty times larger than the timestep implied from stability condition (5.3).

At the end of this subsection, we present the convergence analysis for fully implicit timestepping with the risk adjusted gas spot price following the geometric mean-reverting process (2.10). Table 5.5 shows the convergence results for the following two examples of geometric mean-reverting processes

$$
\begin{aligned}
& d P=3.4(\log 6-\log P) P d t+0.59 P d Z \\
& d P=17.1(\log 3-\log P) P d t+1.33 P d Z
\end{aligned}
$$

where the values of parameters $\sigma$ and $\alpha$ in (5.4) are given in [23]; the parameter values in (5.5) are given in [11]. As expected, for both sets of parameter values, first-order convergence is achieved for both the bang-bang method and the no bang-bang method. 


\begin{tabular}{cccc}
\hline$P$ grid & $I$ grid & \multicolumn{2}{c}{ Timestep size } \\
\cline { 3 - 4 } nodes & nodes & Implicit timestepping & Explicit timestepping \\
\hline 53 & 61 & $6.00 \times 10^{-3}$ & $1.89 \times 10^{-3}$ \\
105 & 121 & $3.00 \times 10^{-3}$ & $4.60 \times 10^{-4}$ \\
209 & 241 & $1.50 \times 10^{-3}$ & $1.14 \times 10^{-4}$ \\
417 & 833 & $7.50 \times 10^{-4}$ & $2.82 \times 10^{-5}$ \\
833 & 961 & $3.75 \times 10^{-4}$ & $7.04 \times 10^{-6}$ \\
\hline
\end{tabular}

TABLE 5.4: Comparison of the timestep size used in our experiment with the maximum timestep size computed from condition (5.3) based on the same mesh discretization. Risk adjusted gas spot price follows the pure mean-reverting process (5.2).

\begin{tabular}{ccc|cc|cc}
\hline P grid & I grid & No. of & \multicolumn{2}{c|}{ Bang-bang method } & \multicolumn{2}{c}{ No bang-bang method } \\
\cline { 4 - 6 } nodes & nodes & timesteps & Value & Ratio & Value & Ratio \\
\hline \multicolumn{7}{c}{ Geometric mean-reverting process (5.4) } \\
\hline 53 & 61 & 500 & 5008430 & n.a. & 5085854 & n.a. \\
105 & 121 & 1000 & 5035055 & n.a. & 5073409 & n.a. \\
209 & 241 & 2000 & 5045524 & 2.54 & 5064888 & 1.46 \\
417 & 481 & 4000 & 5050131 & 2.72 & 5060278 & 1.85 \\
833 & 961 & 8000 & 5052573 & 1.89 & 5057969 & 2.00 \\
\hline \multicolumn{7}{c}{ Geometric mean-reverting process (5.5) } \\
\hline 53 & 61 & 500 & 10123202 & n.a. & 10162773 & n.a. \\
105 & 121 & 1000 & 10168700 & n.a. & 10188203 & n.a. \\
209 & 241 & 2000 & 10188698 & 2.28 & 10198297 & 2.52 \\
417 & 481 & 4000 & 10198143 & 2.12 & 10203148 & 2.08 \\
833 & 961 & 8000 & 10202922 & 1.98 & 10205635 & 1.95 \\
\hline
\end{tabular}

TABLE 5.5: Value of a natural gas storage facility at $P=6 \$ / m m B t u$ and $I=1000$ MMcf. Risk adjusted gas spot price follows the geometric mean-reverting processes (5.4) and (5.5). Convergence ratios are presented for fully implicit timestepping with the bang-bang and the no bang-bang methods. Constant timesteps are used. The payoff function is given in (5.1). Other input parameters are given in Table 5.1. 


\subsection{Incorporating the seasonality effect}

In this subsection, we present numerical results after incorporating the seasonality effect into the equilibrium price of the pure mean-reverting process (5.2). We modified process (5.2) to

$$
d P=2.38(6+\sin (4 \pi t)-P) d t+0.59 P d Z
$$

where the additional term $\sin (4 \pi t)$ makes the equilibrium price a periodic function to represent summer and winter peaks in the equilibrium price. The convergence results for this case are shown in Table 5.6. Comparing Table 5.6 with Table 5.2 indicates that incorporating the seasonality component does not affect the convergence ratio, but does increase the solution value for a fixed refinement level. This is reasonable, since the seasonality effect gives the operator of a gas storage facility an opportunity for obtaining greater profits by using an optimal control strategy that takes advantage of the seasonality feature. For example, a simple strategy of buying and storing gas in spring and then producing and selling gas in summer can normally produce profits from the seasonality effect.

\begin{tabular}{ccc|cc|cc}
\hline$P$ grid & $I$ grid & No. of & \multicolumn{2}{c|}{ Bang-bang method } & \multicolumn{2}{c}{ No bang-bang method } \\
\cline { 4 - 7 } nodes & nodes & timesteps & Value & Ratio & Value & Ratio \\
\hline 53 & 61 & 500 & 4815891 & n.a. & 4889602 & n.a. \\
105 & 121 & 1000 & 4839796 & n.a. & 4875449 & n.a. \\
209 & 241 & 2000 & 4848843 & 2.64 & 4866953 & 1.67 \\
417 & 481 & 4000 & 4853100 & 2.13 & 4862530 & 1.92 \\
833 & 961 & 8000 & 4855485 & 1.78 & 4860397 & 2.07 \\
\hline
\end{tabular}

TABLE 5.6: Value of a natural gas storage facility at $P=6 \$ / m m B t u$ and $I=1000$ MMcf. Risk adjusted gas spot price follows the pure mean-reverting process (5.6) that incorporates the seasonality effect. Convergence ratios are presented for fully implicit timestepping with the bang-bang and the no bang-bang methods. Constant timesteps are used. Payoff function is given in (5.1). Other input parameters are given in Table 5.1.

Figure 5.4 shows the optimal control strategy in the seasonality case that evolves over time as a function of $P$ when the inventory is fixed at $I=1000$ MMcf. From the figure, we can clearly notice the effect of the seasonality on the control strategy: the boundary curve between the zero control region and the negative/positive control region, which represents the control switching boundary between no operation and injecting/producing gas, is periodic when it is far from maturity. Meanwhile, similar to Figure 5.2 for the case without incorporating the seasonality effect, the flat zero control region expands rapidly when the contract is close to maturity.

To further illustrate the difference of the optimal control strategies before and after incorporating the seasonality effect, Figure 5.5 shows the control switching boundary curves at $I=1000$ MMcf as a function of time-to-maturity with respect to processes (5.2) and (5.6), respectively.

\subsection{Incorporating the jump effect}

It is not uncommon to see spot gas price jumps, when gas is used to power electrical generating plants in times of high electricity demand. Spot gas price can jump by as much as $20 \%$ in a single day. To model this effect, in this subsection, we take the pure mean-reverting process (2.8) 


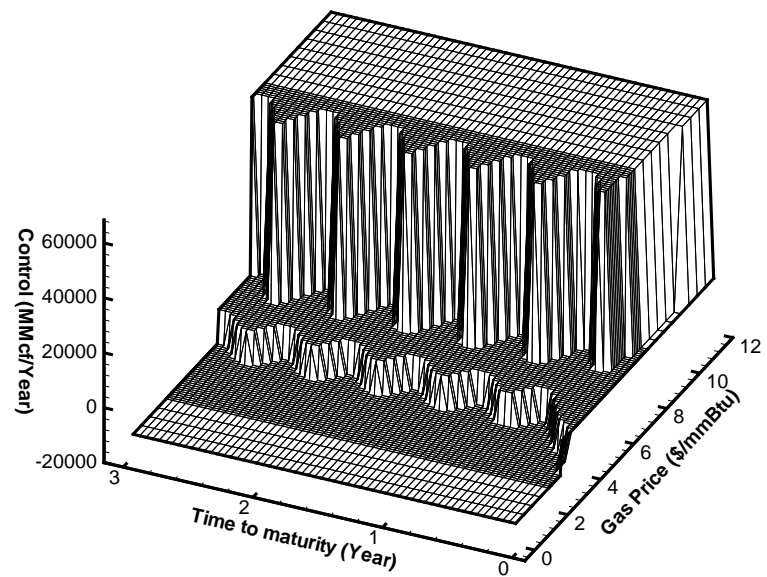

FIGURE 5.4: Optimal control strategy as a function of time-to-maturity $\tau=T-t$ and gas spot price $P$ when the gas inventory resides at $I=1000$ MMcf. Risk adjusted gas spot price follows the pure mean-reverting process (5.6), with seasonality. The payoff function is given in (5.1). Other input parameters are given in Table 5.1. Fully implicit timestepping with the no bang-bang method and with constant timesteps is used.

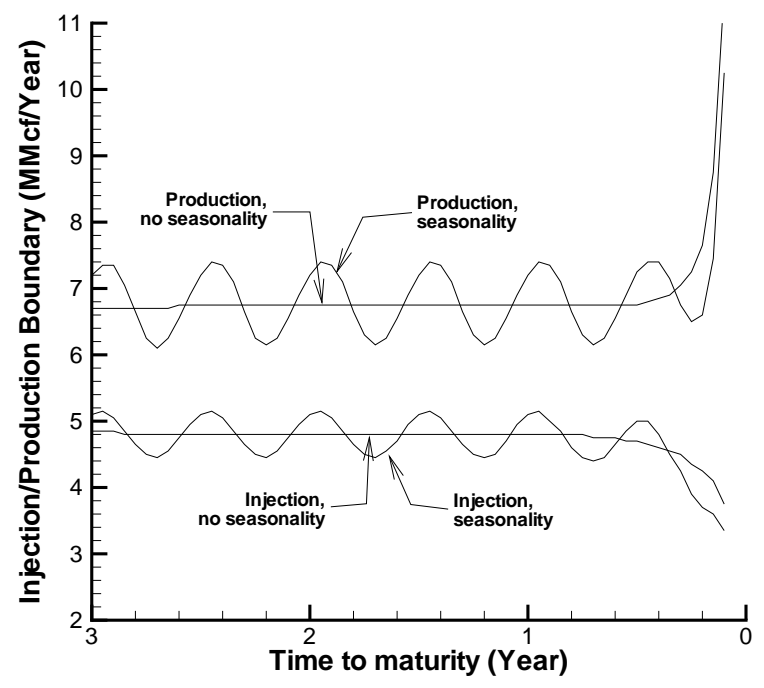

FIGURE 5.5: Control switching boundary curves as a function of time-to-maturity $\tau=T-t$ with respect to processes (5.2) (without incorporating the seasonality effect) and (5.6) (incorporating the seasonality effect) at $I=1000$ MMcf. Payoff function is given in (5.1). Other input parameters are given in Table 5.1. Fully implicit timestepping with the no bang-bang method and with constant timesteps is used. 
and extend it to include a compound Poisson process representing the jump effect, and present numerical results including a jump diffusion process. After adding a jump component, process (2.8) becomes

$$
d P=[\alpha(K(t)-P)-\lambda \kappa P] d t+\sigma P d Z+(\eta-1) P d q,
$$

where

- $d q$ is the independent Poisson process $= \begin{cases}0 & \text { with probability } 1-\lambda d t \\ 1 & \text { with probability } \lambda d t\end{cases}$

- $\lambda$ is the jump intensity representing the mean arrival time of the Poisson process,

- $\eta$ is a random variable representing the jump size of gas price - when $d q=1$, price jumps from $P$ to $P \eta$. We assume that $\eta$ follows a probability density function $g(\eta)$.

- $\kappa$ is $E[\eta-1]$, where $E[\cdot]$ is the expectation operator.

Assuming that the risk adjusted gas spot price follows the jump diffusion process (5.7), the pricing PDE (2.14) turns into the following controlled partial integrodifferential equation (PIDE)

$$
\begin{aligned}
V_{\tau}= & \frac{1}{2} \sigma^{2} P^{2} V_{P P}+[\alpha(K(t)-P)-\lambda \kappa P] V_{P}+\max _{c \in C(I)}\left\{(c-a(c)) P-(c+a(c)) V_{I}\right\} \\
& -r V+\left(\lambda \int_{0}^{\infty} V(P \eta) g(\eta) d \eta-\lambda V\right) .
\end{aligned}
$$

Since there is no control variable in the integral term of PIDE (5.8), we can use the methods described in $[15,16,14]$ to extend the semi-Lagrangian discretization schemes introduced in previous sections to solve the PIDE without difficulty. We note that it is straightforward to use the methods in [15] to show that the resulting scheme is consistent, stable and monotone.

During our numerical experiments, we assume that the probability density function $g(\eta)$ follows log-normal distribution

$$
g(\eta)=\frac{1}{\sqrt{2 \pi} \gamma \eta} \exp \left(-\frac{(\log (\eta)-\nu)^{2}}{2 \gamma^{2}}\right)
$$

with expectation $E[\eta]=\exp \left(\nu+\gamma^{2} / 2\right)$. We will choose values of parameters $\nu$ and $\gamma$ such that $(\eta-1)$, the relative change in the gas spot price, has mean zero and variance 0.04 .

Table 5.7 lists values of the parameters for process (5.7) and for the log-normal density function (5.9), where the parameters of the drift and diffusion components in process (5.7) take the same values as those in process (5.6) for the case without incorporating the jump effect. Note that we set the jump intensity $\lambda=12$ so that random jumps appear approximately once every month. Table 5.8 presents the convergence results for the solution to PIDE (5.8). Table 5.8 shows that the jump effect greatly increases the value of the storage facility.

The results in Table 5.8 also indicate that the no bang-bang method achieves first-order convergence, but not the bang-bang method. In this case, the large value of the jump intensity $\lambda$ causes poor convergence of the bang-bang method. To further study this behavior, in Figure 5.6, we show the control and the value curves at $P=6 \$ / \mathrm{mmBtu}$ as a function of $I$, obtained after the first timestep, on a coarse grid, using these two methods. Figure 5.6 indicates that by actually solving the discrete optimization problem in Algorithm 4.10, the no bang-bang method produces much smoother control and value curves on coarse grids, compared with the bang-bang method. 
Of course, both methods will converge eventually to the same solution. This suggests that the no bang-bang method is a better choice since it appears to converge smoothly at a first-order rate, for cases where the bang-bang method shows somewhat erratic convergence.

\begin{tabular}{cccc}
\hline Parameter & Value & Parameter & Value \\
\hline$\alpha$ & 2.38 & $\nu$ & -0.0196 \\
$K(t)$ & $6+\sin (4 \pi t)$ & $\gamma$ & 0.198 \\
$\sigma$ & 0.59 & $\lambda$ & 12 \\
\hline
\end{tabular}

TABLE 5.7: Input parameters for the jump diffusion process (5.7) and the log-normal density function (5.9). The parameters of the jump size density function are selected so that $E[(\eta-1)]=0$ and $E\left[(\eta-1)^{2}\right]=.04$.

\begin{tabular}{ccc|cc|cc}
\hline$P$ grid & $I$ grid & No. of & \multicolumn{2}{|c|}{ Bang-bang method } & \multicolumn{2}{c}{ No-bang-bang method } \\
\cline { 4 - 7 } nodes & nodes & timesteps & Value & Ratio & Value & Ratio \\
\hline 79 & 61 & 500 & 7995143 & n.a. & 8070698 & n.a. \\
157 & 121 & 1000 & 7962386 & n.a. & 7999775 & n.a. \\
313 & 241 & 2000 & 7951062 & 2.89 & 7971737 & 2.53 \\
625 & 481 & 4000 & 7951032 & 377 & 7961554 & 2.75 \\
1249 & 961 & 8000 & 7951976 & -0.03 & 7957509 & 2.52 \\
\hline
\end{tabular}

TABLE 5.8: Value of a natural gas storage facility at $P=6 \$ / m m B t u$ and $I=1000$ MMcf. Risk adjusted gas spot price follows the pure mean-reverting process (5.7) (incorporating the seasonality and the jump effects). Convergence ratios are presented for fully implicit timestepping with the bang-bang and the no bang-bang methods. Constant timesteps are used. Payoff function is given in (5.1). Input parameters are given in Tables 5.7 and 5.1.

Figure 5.7 compares the control switching boundary curves obtained before and after incorporating the jump effect when $I=1000$ MMcf. The figure indicates that the zero control region (the region contained between two boundary curves) resulting from the jump diffusion process (5.7) is wider than that resulting from process (5.6). This occurs because, under the jump scenario, the operator is willing to wait for a jump in the gas price and then operate the facility after the jump to obtain more profits, which makes the zero control region wider. In addition, Figure 5.7 shows that the jump effect disappears when the contract is close to maturity because of the fear of the revenue loss at maturity due to the payoff structure (5.1).

\section{Conclusion}

In this paper, we develop several implicit timestepping schemes based on a semi-Lagrangian method for the valuation of gas storage contracts. The method has the advantage that it avoids the nonlinear iterations required by an implicit finite difference method and does not introduce extra computational costs. We prove that fully implicit timestepping converges to the viscosity solution of the HJB equation.

We use bang-bang and no bang-bang methods to solve the local optimization problems arising 


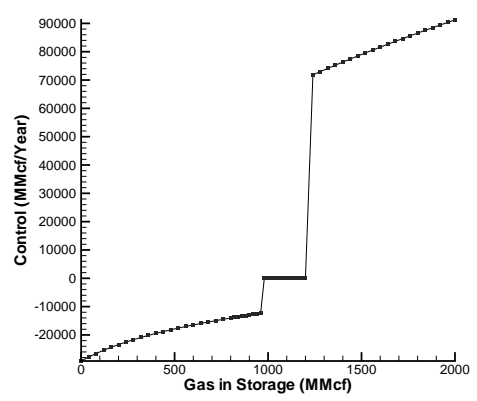

(A) Control curve for the bang-bang method

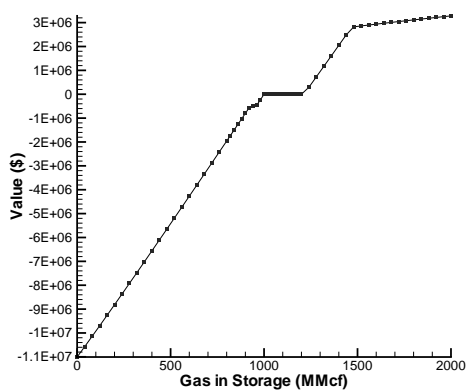

(C) Value curve for the bang-bang method

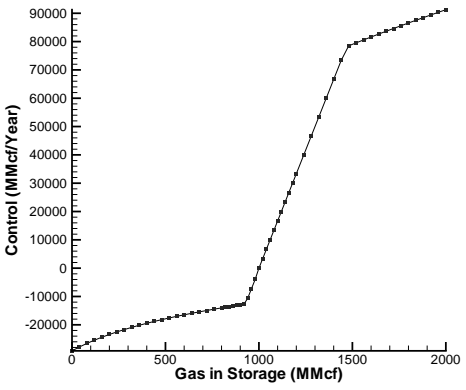

(B) Control curve for the no bang-bang method

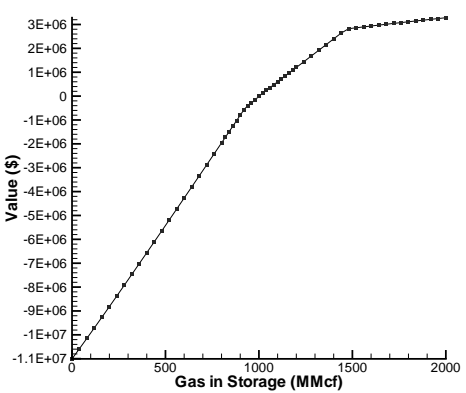

(D) Value curve for the no bang-bang method

Figure 5.6: Control and value curves as a function of gas inventory I obtained after the first timestep with gas price $P=6 \$ / \mathrm{mmBtu}$. Risk adjusted gas spot price follows the pure meanreverting process (5.7). Payoff function is given in (5.1). Input parameters are given in Tables 5.7 and 5.1. Fully implicit timestepping with the no bang-bang method and with constant timesteps is used. 


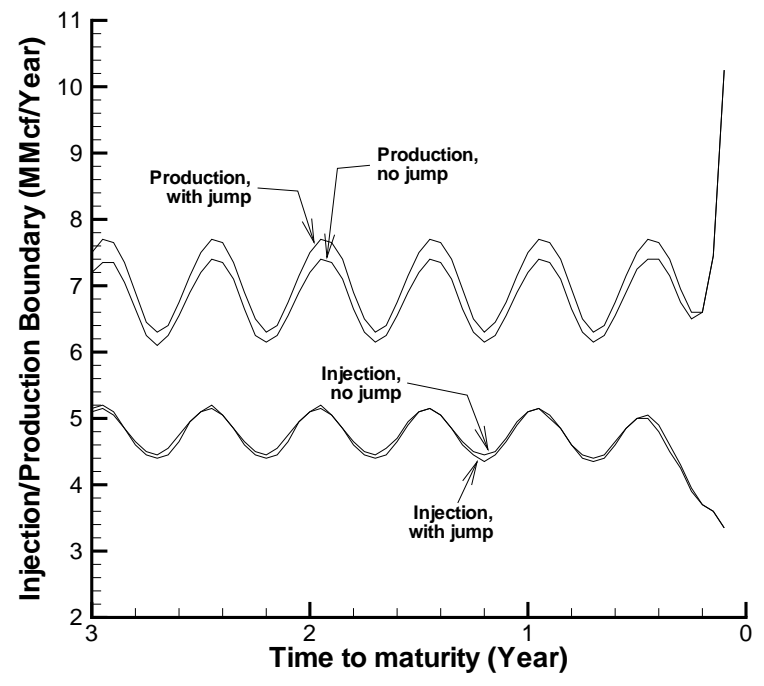

FIGURE 5.7: Control switching boundary curves as a function of time-to-maturity $\tau=T-t$ with respect to processes (5.6) (without incorporating the jump effect) and (5.7) (incorporating the jump effect) at $I=1000 \mathrm{MMcf}$, where parameter values for process (5.7) are given in Table 5.7. Other input parameters are given in Table 5.1. Fully implicit timestepping with the no bang-bang method and with constant timesteps is used.

from the semi-Lagrangian schemes and obtain the optimal operational strategies. The no bangbang method is a more general method and can be applied to solve other problems where the controls are not of bang-bang type.

Our numerical results show that fully implicit and Crank-Nicolson timestepping schemes converge to the same solution. In the case of fully implicit timestepping, the no bang-bang method appears to be superior to the bang-bang method since this method generally exhibits smooth firstorder convergence for cases where we see erratic convergence for the bang-bang method. Numerical results also indicate that Crank-Nicolson timestepping can only achieve at most first-order convergence. Consequently, we recommend the use of fully implicit timestepping, since convergence to the viscosity solution is guaranteed.

In the future, we plan to price gas storage contracts using more realistic gas spot price models, such as regime switching models. We can also apply our schemes to more complex financial contracts that involve more than one stochastic factor and include complicated revenue structures, such as the valuation of gas powered electrical generation plants.

\section{Appendices.}

\section{A Discrete Equation Coefficients}

In this appendix, we give the coefficients of discrete differential operator $\mathcal{L}_{h}$ defined in (3.3). Let $\mu_{i}^{n}=\mu\left(P_{i}, T-\tau^{n}\right)$. For $i=1$, we impose boundary condition (2.18) as $P \rightarrow 0$ by using forward differencing to evaluate the first-order derivative term in (3.1) and setting $V_{P P}=0$, which results 
in

$$
\alpha_{1}^{n}=0 \quad ; \quad \beta_{1}^{n}=\frac{\mu_{1}^{n}}{P_{2}-P_{1}} .
$$

Similarly, for $i=i_{\max }$, the boundary conditions (2.19) as $P \rightarrow \infty$ can be imposed by setting

$$
\alpha_{i_{\max }}^{n}=-\frac{\mu_{i_{\max }}^{n}}{P_{i_{\max }}-P_{i_{\max }-1}} ; \beta_{i_{\max }}^{n}=0
$$

where $\alpha_{i_{\max }}^{n}$ and $\beta_{i_{\max }}^{n}$ are obtained by evaluating the first-order derivative term in (3.1) with backward differencing and setting $V_{P P}=0$.

Away from $i=1$ and $i=i_{\max }$, applying the second-order central differencing in the first and second order derivative terms in equation (3.1) leads to the following values of coefficients $\alpha_{i}^{n}$ and $\beta_{i}^{n}$ :

$$
\begin{aligned}
\alpha_{i, \text { central }}^{n} & =\frac{\sigma^{2} P_{i}^{2}}{\left(P_{i}-P_{i-1}\right)\left(P_{i+1}-P_{i-1}\right)}-\frac{\mu_{i}^{n}}{P_{i+1}-P_{i-1}}, \\
\beta_{i, \text { central }}^{n} & =\frac{\sigma^{2} P_{i}^{2}}{\left(P_{i+1}-P_{i}\right)\left(P_{i+1}-P_{i-1}\right)}+\frac{\mu_{i}^{n}}{P_{i+1}-P_{i-1}}, \quad i=2, \ldots, i_{\max }-1 .
\end{aligned}
$$

If either $\alpha_{i, c e n t r a l}^{n}$ or $\beta_{i, c e n t r a l}^{n}$ is negative, the discrete scheme will not be monotone. Monotonicity can be restored by using first-order forward or backward differencing in the first-order derivative term at the problem nodes. Forward differencing produces:

$$
\begin{aligned}
\alpha_{i, \text { forward }}^{n} & =\frac{\sigma^{2} P_{i}^{2}}{\left(P_{i}-P_{i-1}\right)\left(P_{i+1}-P_{i-1}\right)}, \\
\beta_{i, \text { forward }} & =\frac{\sigma^{2} P_{i}^{2}}{\left(P_{i+1}-P_{i}\right)\left(P_{i+1}-P_{i-1}\right)}+\frac{\mu_{i}^{n}}{P_{i+1}-P_{i}}, \quad i=2, \ldots, i_{\max }-1 .
\end{aligned}
$$

while backward differencing gives:

$$
\begin{aligned}
\alpha_{i, \text { backward }}^{n} & =\frac{\sigma^{2} P_{i}^{2}}{\left(P_{i}-P_{i-1}\right)\left(P_{i+1}-P_{i-1}\right)}-\frac{\mu_{i}^{n}}{P_{i}-P_{i-1}}, \\
\beta_{i, \text { backward }}^{n} & =\frac{\sigma^{2} P_{i}^{2}}{\left(P_{i+1}-P_{i}\right)\left(P_{i+1}-P_{i-1}\right)}, \quad i=2, \ldots, i_{\max }-1 .
\end{aligned}
$$

On one hand, we want to use central differencing to achieve second-order correctness. On the other hand, we need to maintain $\alpha_{i}^{n}$ and $\beta_{i}^{n}$ positive so that the scheme is monotone. Consequently, we decide a central or forward/backward discretization at each node $\left(P_{i}, I_{j}\right), i=2, \ldots, i_{\max }-1$, $j=1, \ldots, j_{\max }$, based on the following strategy: 


$$
\begin{aligned}
& \text { If }\left[\alpha_{i, \text { central }}^{n} \geq 0 \text { and } \beta_{i, \text { central }}^{n} \geq 0\right] \text { then } \\
& \quad \begin{array}{l}
\alpha_{i}^{n}=\alpha_{i, \text { central }}^{n} \\
\beta_{i}^{n}=\beta_{i, \text { central }}^{n}
\end{array} \\
& \text { ElseIf }\left[\beta_{i, \text { forward }}^{n} \geq 0\right] \text { then } \\
& \alpha_{i}^{n}=\alpha_{i, \text { forward }}^{n} \\
& \beta_{i}^{n}=\beta_{i, \text { forward }}^{n} \\
& \text { Else } \\
& \alpha_{i}^{n}=\alpha_{i, \text { backward }}^{n} \\
& \beta_{i}^{n}=\beta_{i, \text { backward }}^{n} \\
& \text { EndIf }
\end{aligned}
$$

Note that (A.1-A.2) and (A.6) guarantee that both $\alpha_{i}^{n}$ and $\beta_{i}^{n}$ are non-negative. Equation (2.13) implies that $\mu_{i}^{n}$ will be small if $P_{i}$ is close to $K\left(T-\tau^{n}\right)$, which is the equilibrium price of the risk adjusted natural gas spot price at $t=T-\tau^{n}$. In particular, if there exists a node $P_{i}$ such that $P_{i}=K\left(T-\tau^{n}\right)$, then we have $\mu_{i}^{n}=0$ from (2.13). For small values of $\mu_{i}^{n}$, equation (A.3) imply that $\alpha_{i, \text { central }}^{n}$ and $\beta_{i, c e n t r a l}^{n}$ are positive. Hence the strategy (A.6) will use central differencing for those nodes $P_{i}$ close to the equilibrium price. These nodes are in the region of interest, since the gas spot price will not stray too far away from the mean-reversion equilibrium price. Consequently, the use of a low-order differencing scheme for nodes far away from the equilibrium price should not result in poor convergence for the nodes near the equilibrium price. We will verify this in our numerical experiments.

\section{B Discrete Optimal Control Strategy}

In this Appendix, we derive a discretization based on purely physical reasoning, assuming that the operator of a gas storage facility can change the controls only at fixed discrete times. We will see that the final discrete equations are identical to the fully implicit semi-Lagrangian discretization derived in Section 3.1. This provides justification for the choice of integration points used in deriving the fully implicit discretization (3.14-3.15). It is interesting to observe that the fully implicit semi-Lagrangian discretization can be interpreted as a no-arbitrage jump condition.

Suppose we have $N+1$ discrete times, denoted by $0=t^{0}<t^{1}<\ldots<t^{N}=T$, over the period from $t=0$ to $t=T$. Let $t^{0}, t^{1}, \ldots, t^{N-1}$ be the discrete operation times where the operator of a natural gas storage facility can choose to change the controls. At each decision time $t=t^{n-1}$, $n=1,2, \ldots, N$, the operator either does nothing or chooses to inject or produce gas at a constant rate. This decision cannot be changed until the next decision time $t^{n}$.

We assume that no cash flows will occur until the end of a time interval. In other words, given a decision at $t^{n-1}$, then the revenue is obtained for all the gas produced in $\left[t^{n-1}, t^{n}\right)$ at $t^{n}$. We also imagine that the inventory is regarded as constant during $\left[t^{n-1}, t^{n}\right)$. The inventory is adjusted only at $t^{n}$ to reflect the gas produced/injected during $\left[t^{n-1}, t^{n}\right)$. Clearly, if we let $N \rightarrow \infty$, then the above discrete control scenario will turn into a continuous control scenario.

Let us consider a path of inventory level in the gas storage facility at different times with respect to a fixed natural gas spot price, denoted by $\hat{\mathcal{I}}(P, t)$. Such a path is generated by the 
control strategy that the operator applies on the storage facility along a specific time path. ${ }^{1}$ As described above, under our discrete control scenario, the gas inventory changes only at discrete times $t^{n}$. Thus, the path $\hat{\mathcal{I}}(P, t)$ is a piece-wise constant function with $\hat{\mathcal{I}}(P, t)=\hat{\mathcal{I}}\left(P, t^{n-1}\right)$ for $t \in\left[t^{n-1}, t^{n}\right), n=1,2, \ldots, N$. Let $\hat{V}(P, \hat{\mathcal{I}}(P, t), t)$ denote the value of the gas storage facility at forward time $t$ with gas spot price fixed at $P$ and gas inventory following the path $\hat{\mathcal{I}}(P, t)$. In the rest of this section, we will investigate the value of $\hat{V}(P, \hat{\mathcal{I}}(P, t), t)$ at discrete times $t=t^{n-1}$, $n=1,2, \ldots, N .^{2}$ Note that in contrast to the inventory path $\hat{\mathcal{I}}$, we use notation $I$ to represent a gas inventory value independent of $P$ and $t$, as appears in PDE (2.14).

We denote by $\hat{\zeta}\left(P, \hat{\mathcal{I}}\left(P, t^{n-1}\right), t^{n-1}\right)$ the constant rate of the control chosen by the storage facility operator at $t=t^{n-1}$ when the gas spot price is fixed at $P$ and gas inventory path $\hat{\mathcal{I}}$ arrives at $\hat{\mathcal{I}}\left(P, t^{n-1}\right)$. The control $\hat{\zeta}\left(P, \hat{\mathcal{I}}\left(P, t^{n-1}\right), t^{n-1}\right)$ is applied to the gas storage facility for $t \in$ $\left[t^{n-1}, t^{n}\right)$. In accordance with the convention in Section 2.1, we assume that $\hat{\zeta}\left(P, \hat{\mathcal{I}}\left(P, t^{n-1}\right), t^{n-1}\right)>$ 0 represents production, $\hat{\zeta}\left(P, \hat{\mathcal{I}}\left(P, t^{n-1}\right), t^{n-1}\right)<0$ represents injection, and $\hat{\zeta}\left(P, \hat{\mathcal{I}}\left(P, t^{n-1}\right), t^{n-1}\right)=$ 0 represents no operation. From Section 2.1, we require the control $\hat{\zeta}$ to satisfy the constraint $\hat{\zeta}\left(P, \hat{\mathcal{I}}\left(P, t^{n-1}\right), t^{n-1}\right) \in C\left(\hat{\mathcal{I}}\left(P, t^{n-1}\right)\right)$, where $C(\hat{\mathcal{I}})=\left[c_{\min }(\hat{\mathcal{I}}),-k_{5}\right] \cup\left[0, c_{\max }(\hat{\mathcal{I}})\right]$, as defined in equation (2.7).

According to the above assumptions, the gas inventory along the path $\hat{\mathcal{I}}$ is fixed at $\hat{\mathcal{I}}\left(P, t^{n-1}\right)$ for $t \in\left[t^{n-1}, t^{n}\right)$, and switches to $\hat{\mathcal{I}}\left(t^{n}\right)$ at $t=t^{n}$. Let $\Delta t^{n-1}=t^{n}-t^{n-1}$. The change of the gas inventory from $t=t^{n-1}$ to $t=t^{n}$, due to gas injection, production and gas loss, satisfies

$$
\hat{\mathcal{I}}\left(P, t^{n}\right)=\hat{\mathcal{I}}\left(P, t^{n-1}\right)-\Delta t^{n-1}\left[\hat{\zeta}\left(P, \hat{\mathcal{I}}\left(P, t^{n-1}\right), t^{n-1}\right)+a\left(\hat{\zeta}\left(P, \hat{\mathcal{I}}\left(P, t^{n-1}\right), t^{n-1}\right)\right)\right] .
$$

Note that equation (B.1) is the discrete-time version of the ODE (2.4) with variable $c$ replaced by $\hat{\zeta}$. For convenience, we introduce the notation $\hat{\mathcal{I}}^{n}=\hat{\mathcal{I}}\left(P, t^{n}\right), \hat{\zeta}^{n}=\hat{\zeta}\left(P, \hat{\mathcal{I}}\left(P, t^{n}\right), t^{n}\right)$. We drop the dependence of $\hat{\mathcal{I}}$ on $P$ and that of $\hat{\zeta}$ on $(P, \hat{\mathcal{I}})$ for the purpose of simplifying our presentation. Using the notation introduced above, equation (B.1) can be written as

$$
\hat{\mathcal{I}}^{n}=\hat{\mathcal{I}}^{n-1}-\Delta t^{n-1}\left(\hat{\zeta}^{n-1}+a\left(\hat{\zeta}^{n-1}\right)\right) .
$$

Due to physical storage constraints, after imposing the control $\hat{\zeta}^{n-1}$ at $t=t^{n-1}$, the resulting gas inventory $\hat{\mathcal{I}}^{n}$ from (B.2) must remain inside the domain $\left[0, I_{\max }\right]$. In particular, if $\hat{\mathcal{I}}^{n-1}=0$, then the operator cannot produce gas at $t=t^{n-1}$ and thus we require $\hat{\zeta}^{n-1}+a\left(\hat{\zeta}^{n-1}\right) \leq 0$. Similarly, if $\hat{\mathcal{I}}^{n-1}=I_{\max }$, we require $\hat{\zeta}^{n-1}+a\left(\hat{\zeta}^{n-1}\right) \geq 0$. These two conditions are the discrete version of boundary conditions (2.16) and (2.17) satisfied by PDE (2.14), respectively. We refer to a control $\hat{\zeta}^{n-1}$ as admissible control if it satisfies $\hat{\zeta}^{n-1} \in C\left(\hat{\mathcal{I}}^{n-1}\right)$ and $\hat{\mathcal{I}}^{n}$ calculated from (B.2) is bounded within $\left[0, I_{\max }\right]$. We denote by $\hat{C}^{n-1}$ the set of all admissible controls at $t=t^{n-1}$.

Recall that, under the discrete control assumptions, for $t \in\left[t^{n-1}, t^{n}\right)$, the gas inventory is fixed at $\hat{\mathcal{I}}^{n-1}$ and no cash-flows appear. At $t=t^{n}$, the inventory switches to $\hat{\mathcal{I}}^{n}$ and a revenue $\Delta t^{n-1}\left(\hat{\zeta}^{n-1}-a\left(\hat{\zeta}^{n-1}\right)\right) P$ is created, which is the product of the amount of gas lost, injected or produced, i.e., $\Delta t^{n-1}\left(\hat{\zeta}^{n-1}-a\left(\hat{\zeta}^{n-1}\right)\right)$, during the period of $\left[t^{n-1}, t^{n}\right)$ and the gas spot price. Let

\footnotetext{
${ }^{1}$ We do not need to introduce the control as a variable in $\hat{\mathcal{I}}$ because we assume that an optimal control strategy will always be chosen by a rational operator, as explained below, under our discrete control scenario; the optimal strategy will produce an optimal control that is determined by the gas spot price $P$ and gas inventory $\hat{\mathcal{I}}$ at the decision time. Hence, we will not set control as a variable in $\hat{\mathcal{I}}$.

${ }^{2}$ Note that $\hat{V}(P, \hat{\mathcal{I}}(P, t), t)$ for $t=t^{N}$ is given by a payoff condition.
} 
$t^{n-}=t^{n}-\epsilon$ with $\epsilon>0, \epsilon \rightarrow 0$, that is, $t^{n-}$ is the time infinitesimally before $t^{n}$. By no-arbitrage [37], at $t=t^{n}, \hat{V}$ must satisfy the following condition

$$
\hat{V}\left(P, \hat{\mathcal{I}}^{n}, t^{n}\right)=\hat{V}\left(P, \hat{\mathcal{I}}^{n-1}, t^{n-}\right)-\Delta t^{n-1}\left(\hat{\zeta}^{n-1}-a\left(\hat{\zeta}^{n-1}\right)\right) P .
$$

Out of a variety of choices, the problem next is to find the optimal value of $\hat{\zeta}^{n-1}$. A rational operator will choose a control $\hat{\zeta}^{n-1}$ that maximizes the facility value $\hat{V}$ at $t=t^{n-}$. Thus, the no-arbitrage condition (B.3) can be written as

$$
\begin{gathered}
\hat{V}\left(P, \hat{\mathcal{I}}^{n-1}, t^{n-}\right)=\max _{\hat{\zeta}^{n-1} \in \hat{C}^{n-1}}\left\{\hat{V}\left(P, \hat{\mathcal{I}}^{n}, t^{n}\right)+\Delta t^{n-1}\left(\hat{\zeta}^{n-1}-a\left(\hat{\zeta}^{n-1}\right)\right) P\right\}, \\
\hat{\mathcal{I}}^{n}=\hat{\mathcal{I}}^{n-1}-\Delta t^{n-1}\left(\hat{\zeta}^{n-1}+a\left(\hat{\zeta}^{n-1}\right)\right) ; \hat{\mathcal{I}}^{n} \in\left[0, I_{\text {max }}\right] \text { for any } \hat{\zeta}^{n-1} \in \hat{C}^{n-1} .
\end{gathered}
$$

During the interval $\left[t^{n-1}, t^{n-}\right]$, the inventory in the path $\hat{\mathcal{I}}$ is a constant $\hat{\mathcal{I}}^{n-1}$ and no cash flows occur, hence the value of the facility is given by the solution of the PDE

$$
\hat{V}_{t}+\frac{1}{2} \sigma^{2} P^{2} \hat{V}_{P P}+\mu(P, t) \hat{V}_{P}-r \hat{V}=0 .
$$

We can semidiscretize this in time over the interval $\left[t^{n-1}, t^{n-}\right]$ using fully implicit timestepping

$$
\begin{gathered}
\frac{\hat{V}\left(P, \hat{\mathcal{I}}^{n-1}, t^{n-}\right)-\hat{V}\left(P, \hat{\mathcal{I}}^{n-1}, t^{n-1}\right)}{\Delta t^{n-1}}+\frac{\sigma^{2} P^{2}}{2} \hat{V}_{P P}\left(P, \hat{\mathcal{I}}^{n-1}, t^{n-1}\right) \\
+\mu\left(P, t^{n-1}\right) \hat{V}_{P}\left(P, \hat{\mathcal{I}}^{n-1}, t^{n-1}\right)-r \hat{V}\left(P, \hat{\mathcal{I}}^{n-1}, t^{n-1}\right)=0 .
\end{gathered}
$$

Note that we will solve equation (B.5) backward in time, so that fully implicit timestepping uses the values of the diffusion, drift and discounting terms at $t=t^{n-1}$.

Substituting equation (B.4) into equation (B.6) gives

$$
\begin{aligned}
& \frac{\max _{\hat{\zeta}^{n-1} \in \hat{C}^{n-1}}\left\{\hat{V}\left(P, \hat{\mathcal{I}}^{n}, t^{n}\right)+\Delta t^{n-1}\left(\hat{\zeta}^{n-1}-a\left(\hat{\zeta}^{n-1}\right)\right) P\right\}-\hat{V}\left(P, \hat{\mathcal{I}}^{n-1}, t^{n-1}\right)}{\Delta t^{n-1}} \\
& +\frac{\sigma^{2} P^{2}}{2} \hat{V}_{P P}\left(P, \hat{\mathcal{I}}^{n-1}, t^{n-1}\right)+\mu\left(P, t^{n-1}\right) \hat{V}_{P}\left(P, \hat{\mathcal{I}}^{n-1}, t^{n-1}\right)-r \hat{V}\left(P, \hat{\mathcal{I}}^{n-1}, t^{n-1}\right) \\
& =0,
\end{aligned}
$$

where

$$
\hat{\mathcal{I}}^{n}=\hat{\mathcal{I}}^{n-1}-\Delta t^{n-1}\left(\hat{\zeta}^{n-1}+a\left(\hat{\zeta}^{n-1}\right)\right) ; \hat{\mathcal{I}}^{n} \in\left[0, I_{\max }\right] \text { for any } \hat{\zeta}^{n-1} \in \hat{C}^{n-1} .
$$

Based on the semidiscretization scheme (B.7), assuming $\hat{V}\left(P, \hat{\mathcal{I}}^{n}, t^{n}\right)$ is known, we are able to compute $\hat{V}\left(P, \hat{\mathcal{I}}^{n-1}, t^{n-1}\right)$ at the previous time $t=t^{n-1}$ with gas inventory moving along a path (given in (B.8)) generated by the optimal control strategy.

Equation (B.7) is written in terms of forward times $t$. We can rewrite this equation in terms of backward times $\tau=T-t$. Let $\mathcal{I}(P, \tau)$ denote the gas inventory path at backward times as a function of $P$ and $\tau=T-t$, that is, $\mathcal{I}(P, \tau)=\mathcal{I}(P, T-t)=\hat{\mathcal{I}}(P, t)$. Let $\zeta(P, \mathcal{I}(P, \tau), \tau)$ denote the control at backward times as a function of $P, \mathcal{I}, \tau$ with $\zeta(P, \mathcal{I}(P, \tau), \tau)=\zeta(P, \mathcal{I}(P, T-t), T-$ $t)=\hat{\zeta}(P, \hat{\mathcal{I}}(P, t), t)$. Similarly, we can define the value of a gas storage facility as a function of $P, \mathcal{I}, \tau$ as $V(P, \mathcal{I}(P, \tau), \tau)$, where, in terms of $\hat{V}(P, \hat{\mathcal{I}}, t)$, we have the identity $V(P, \mathcal{I}(P, \tau), \tau)=$ $V(P, \mathcal{I}(P, T-t), T-t)=\hat{V}(P, \hat{\mathcal{I}}(P, t), t)$. Let $k=N-n, k=0,1, \ldots, N-1$, so that $k$ counts 
backwards. Since $t^{N}=T$, we have that $\tau^{k}=T-t^{n}, \tau^{k+1}=T-t^{n-1}$. Let $\mathcal{I}^{k}=\mathcal{I}\left(P, \tau^{k}\right)$, $\zeta^{k}=\zeta\left(P, \mathcal{I}\left(P, \tau^{k}\right), \tau^{k}\right), \Delta \tau^{k}=\tau^{k+1}-\tau^{k}{ }^{3}$ We can obtain the following identities between discrete forward and backward times:

$$
\begin{gathered}
\hat{\zeta}^{n-1}=\zeta^{k+1} ; \quad \hat{\mathcal{I}}^{n-1}=\mathcal{I}^{k+1} ; \quad \hat{\mathcal{I}}^{n}=\mathcal{I}^{k} ; \quad \Delta t^{n-1}=\Delta \tau^{k} ; \quad \mu\left(P, t^{n-1}\right)=\mu\left(P, T-\tau^{k+1}\right) \\
\hat{V}\left(P, \hat{\mathcal{I}}^{n-1}, t^{n-1}\right)=V\left(P, \mathcal{I}^{k+1}, \tau^{k+1}\right) ; \quad \hat{V}\left(P, \hat{\mathcal{I}}^{n}, t^{n}\right)=V\left(P, \mathcal{I}^{k}, \tau^{k}\right) .
\end{gathered}
$$

Using identities of (B.9) in equations (B.7-B.8), we obtain the optimal control problem in backwards time

$$
\begin{aligned}
& \frac{\left.V\left(P, \mathcal{I}^{k+1}, \tau^{k+1}\right)-\max _{\zeta^{k+1} \in C^{k+1}}\left\{V\left(P, \mathcal{I}^{k}, \tau^{k}\right)+\Delta \tau^{k}\left(\zeta^{k+1}-a\left(\zeta^{k+1}\right)\right) P\right)\right\}}{\Delta \tau^{k}} \\
& \quad=\frac{\sigma^{2} P^{2}}{2} V_{P P}\left(P, \mathcal{I}^{k+1}, \tau^{k+1}\right)+\mu\left(P, T-\tau^{k+1}\right) V_{P}\left(P, \mathcal{I}^{k+1}, \tau^{k+1}\right)-r V\left(P, \mathcal{I}^{k+1}, \tau^{k+1}\right)
\end{aligned}
$$

where

$$
\mathcal{I}^{k}=\mathcal{I}^{k+1}-\Delta \tau^{k}\left(\zeta^{k+1}+a\left(\zeta^{k+1}\right)\right) ; C^{k+1} \subseteq C\left(\mathcal{I}^{k+1}\right), \mathcal{I}^{k} \in\left[0, I_{\max }\right] \text { for any } \zeta^{k+1} \in C^{k+1} .
$$

Let us further discretize the scheme (B.11-B.10) in the $(P, \mathcal{I})$ direction by setting

$$
\begin{gathered}
k=n, \quad P=P_{i}, \quad \mathcal{I}^{k+1}=I_{j}, \quad \zeta^{k+1}=\zeta_{i, j}^{n+1}, \quad C^{k+1}=C_{j}^{n+1}, \\
V\left(P, \mathcal{I}^{k+1}, \tau^{k+1}\right)=V_{i, j}^{n+1}, \quad V\left(P, \mathcal{I}^{k}, \tau^{k}\right)=V_{i, j(i, n+1)}^{n}
\end{gathered}
$$

in (B.11-B.10) and replacing the right hand side of (B.10) by the discrete differential operator $\left(\mathcal{L}_{h} V\right)_{i, j}^{n+1}$ given in (3.3). Then we can recognize that the full discretization of the semi-discretization (B.11-B.10) (based on a discrete optimal control) is algebraically identical to the semi-Lagrangian, fully implicit discretization (3.14-3.15).

\section{Solution of the Local Optimization Problems}

According to Algorithm 4.10, we need to solve a constrained optimization problem

$$
\begin{aligned}
\max _{\left(\zeta_{i, j}^{n}, \zeta_{i, j}^{n+1}\right) \in C_{j}^{n+1}}\{ & V_{i, j(i, n+1)}^{n}+(1-\theta) \Delta \tau^{n}\left(\zeta_{i, j}^{n+1}-a\left(\zeta_{i, j}^{n+1}\right)\right) P_{i}+ \\
& \left.\theta \Delta \tau^{n}\left(\zeta_{i, j}^{n}-a\left(\zeta_{i, j}^{n}\right)\right) P_{i}+\theta \Delta \tau^{n}\left(\mathcal{L}_{h} V\right)_{i, j(i, n+1)}^{n}\right\}, \\
\text { with } \mathcal{I}_{j(i, n+1)}^{n}= & I_{j}-(1-\theta) \Delta \tau^{n}\left(\zeta_{i, j}^{n+1}+a\left(\zeta_{i, j}^{n+1}\right)\right)-\theta \Delta \tau^{n}\left(\zeta_{i, j}^{n}+a\left(\zeta_{i, j}^{n}\right)\right)
\end{aligned}
$$

at every mesh node $\left(P_{i}, I_{j}\right)$ and at every discrete timestep $\tau^{n}$. In this section, we give an overview of the bang-bang and no bang-bang methods for solving problem (C.1). We omit the algorithm details due to limitation of space.

\footnotetext{
${ }^{3}$ Here we also drop the dependence of $\mathcal{I}$ on $P$ and that of $\zeta$ on $(P, \mathcal{I})$ for convenience.
} 


\section{No bang-bang method}

Problem (C.1) is nonlinear since the admissible set $C_{j}^{n+1}$ for controls depends on the value of $\mathcal{I}_{j(i, n+1)}^{n}$, which in turn is a function of controls. This makes it difficult to directly solve problem (C.1), especially in the case of Crank-Nicolson timestepping.

We simplify the problem by changing unknowns from $\zeta_{i, j}^{n}, \zeta_{i, j}^{n+1}$ to $\mathcal{I}_{j(i, n+1)}^{n}$. Specifically, we can write equation (C.2) as

$$
(1-\theta) \Delta \tau^{n} \zeta_{i, j}^{n+1}+\theta \Delta \tau^{n} \zeta_{i, j}^{n}=\left(I_{j}-\mathcal{I}_{j(i, n+1)}^{n}\right)-(1-\theta) \Delta \tau^{n} a\left(\zeta_{i, j}^{n+1}\right)-\theta \Delta \tau^{n} a\left(\zeta_{i, j}^{n}\right) .
$$

Substituting equation (C.3) into (C.1) leads to

$$
\begin{aligned}
\max _{\left(\zeta_{i, j}^{n}, \zeta_{i, j}^{n+1}\right) \in C_{j}^{n+1}}\{ & V_{i, j(i, n+1)}^{n}-\mathcal{I}_{j(i, n+1)}^{n} P_{i}+\theta \Delta \tau^{n}\left(\mathcal{L}_{h} V\right)_{i, j(i, n+1)}^{n} \\
& \left.-2(1-\theta) \Delta \tau^{n} a\left(\zeta_{i, j}^{n+1}\right) P_{i}-2 \theta \Delta \tau^{n} a\left(\zeta_{i, j}^{n}\right) P_{i}\right\}+I_{j} P_{i}
\end{aligned}
$$

In the following we consider Crank-Nicolson timestepping $(\theta=1 / 2)$ in (C.4); the same method can be applied to fully implicit timestepping, which is a much easier problem compared to CrankNicolson timestepping. Since $a\left(\zeta_{i, j}^{n+1}\right)$ and $a\left(\zeta_{i, j}^{n}\right)$ are either 0 or $k_{5}$ according to the signs of $\zeta_{i, j}^{n+1}$ and $\zeta_{i, j}^{n}$, we can drop the dependence of the objective function in (C.4) on $\zeta_{i, j}^{n+1}, \zeta_{i, j}^{n}$ by separately considering four regions corresponding to the different combinations of signs of $\zeta_{i, j}^{n+1}$ and $\zeta_{i, j}^{n}$. For example, one region is $\left(\zeta_{i, j}^{n+1}, \zeta_{i, j}^{n}\right) \in\left[0, c_{\max }\left(I_{j}\right)\right] \times\left[0, c_{\max }\left(I_{j(i, n+1)}^{n}\right)\right]$; when $\zeta_{i, j}^{n+1}, \zeta_{i, j}^{n}$ lie in this region we have $a\left(\zeta_{i, j}^{n+1}\right)=a\left(\zeta_{i, j}^{n}\right)=0$. Meanwhile, for all controls $\zeta_{i, j}^{n+1}, \zeta_{i, j}^{n}$ in any of the four regions, it can be shown that the corresponding values of $\mathcal{I}_{j(i, n+1)}^{n}$ consist of an interval $\left[I_{\min }^{n}, I_{\max }^{n}\right] \subseteq\left[0, I_{\max }\right]$, where the bounds $I_{\min }^{n}$ and $I_{\max }^{n}$ are independent of controls $\zeta_{i, j}^{n+1}$ and $\zeta_{i, j}^{n}$. Therefore, through the changing of unknowns, instead of solving one two-dimensional nonlinear optimization problem (C.1), identically, we can solve four one-dimensional optimization problems and pick the maximum value among the four; each of the optimization problems has the form

$$
\begin{array}{r}
\max _{\mathcal{I}_{j(i, n+1)}^{n} \in\left[I_{\min }^{n}, I_{\max }^{n}\right]}\left\{V_{i, j(i, n+1)}^{n}-\mathcal{I}_{j(i, n+1)}^{n} P_{i}+\theta \Delta \tau^{n}\left(\mathcal{L}_{h} V\right)_{i, j(i, n+1)}^{n}\right\} \\
-2(1-\theta) \Delta \tau^{n} D^{n+1} P_{i}-2 \theta \Delta \tau^{n} D^{n} P_{i}+I_{j} P_{i}
\end{array}
$$

where $D^{n}, D^{n+1}$ are constants determined by the signs of $\zeta_{i, j}^{n}$ and $\zeta_{i, j}^{n+1}$.

As explained in Section 3, the quantities of the form $(\cdot)_{i, j(i, n+1)}^{n}$ in (C.5) are obtained from an interpolation method. If linear interpolation is used, then the optimal value of $\mathcal{I}_{j(i, n+1)}^{n}$ for problem (C.5) resides either at boundaries $I_{\min }^{n}, I_{\max }^{n}$ or at grid nodes lying between $I_{\min }^{n}$ and $I_{\max }^{n}$. As a result, we only need to check these discrete nodes and return the maximum value as the solution to problem (C.5).

If quadratic interpolation is used, then we divide interval $\left[I_{\min }^{n}, I_{\max }^{n}\right]$ into several sub-intervals; within each sub-interval, the same interpolation stencils are used to compute quantities $(\cdot)_{i, j(i, n+1)}^{n}$. For each sub-interval, we calculate the maximum value of the objective function in (C.5) with $\mathcal{I}_{j(i, n+1)}^{n}$ residing inside that sub-interval. Finally, we compare the values calculated for all subintervals and select the maximum as the solution to problem (C.5). To reduce the effect of nonmonotonicity caused by quadratic interpolation, we limit the interpolation by requiring [39, 9] 
(assuming $\left.I_{s} \leq \mathcal{I}_{j(i, n+1)}^{n} \leq I_{s+1}\right)$

$$
V_{i, j(i, n+1)}^{n} \leq \max \left\{V_{i, s}^{n}, V_{i, s+1}^{n}\right\} \quad ; \quad V_{i, j(i, n+1)}^{n} \geq \min \left\{V_{i, s}^{n}, V_{i, s+1}^{n}\right\} .
$$

In the case of fully implicit timestepping, after obtaining the optimal $\mathcal{I}_{j(i, n+1)}^{n}$ for problem (C.1), we can compute the optimal control $\zeta_{i, j}^{n+1}$ from equation (C.3). In this case, the variables $\mathcal{I}_{j(i, n+1)}^{n}$ and $\zeta_{i, j}^{n+1}$ have physical meanings. Recall the discrete optimal control scenario described in Appendix B. Under this scenario, $I_{j}$ and $\mathcal{I}_{j(i, n+1)}^{n}$ represent the gas inventory at the forward times $t=t^{k}$ and $t=t^{k+1}$, respectively, where $t^{k}=T-\tau^{n+1} ; \zeta_{i, j}^{n+1}$ represent the optimal operation that the operator imposes on the storage facility during the interval $\left[t^{k}, t^{k+1}\right)$. In the case of CrankNicolson timestepping, although we can solve problem (C.1), given the optimal value of $\mathcal{I}_{j(i, n+1)}^{n}$, we cannot uniquely determine the values of the control variables $\zeta_{i, j}^{n}$ and $\zeta_{i, j}^{n+1}$ from (C.3). Nor do these variables have simple physical meanings.

Remark C.1. When incorporating nonlinear revenue structures such as that given in [35], the control for the resulting equation is not of bang-bang type. Nevertheless, the above method can still be used to solve the optimization problem in the case of fully implicit timestepping $(\theta=0)$. Thus, the fully implicit semi-Lagrangian discreteization is applicable to a wide range of HJB equations including those that inherit the no bang-bang control feature.

\section{Bang-bang method}

As shown in [32], the optimization problem

$$
\max _{c \in C(I)}\left\{(c-a(c)) P-(c+a(c)) V_{I}\right\}
$$

in PDE (2.14) exhibits a bang-bang control feature. Specifically, the optimal value for $c$ in (C.7) is either $c_{\max }(I), c_{\min }(I)$, or 0. Similarly, we can apply the bang-bang control method to problem (C.1) since the semi-Lagrangian discretization (3.18) converges to the unique viscosity solution of PDE (2.14).

In fact, consider fully implicit timestepping $(\theta=0)$ in (C.1), assuming that linear interpolation is used for $V_{i, j(i, n+1)}^{n}$ and $\Delta \tau^{n}$ is sufficiently small. In this case, Lemma D.1 implies that $\zeta_{i, j}^{n+1} \in C\left(I_{j}\right)$. From equation (C.2), the maximum value of $\mathcal{I}_{j(i, n+1)}^{n}$, attained by choosing $\zeta_{i, j}^{n+1}=c_{\min }\left(I_{j}\right)$, resides between grid nodes $I_{j}$ and $I_{j+1}$, provided that $\Delta \tau^{n}$ is sufficiently small. Similarly, if $\Delta \tau^{n}$ is small enough, the minimum value of $\mathcal{I}_{j(i, n+1)}^{n}$, obtained by taking $\zeta_{i, j}^{n+1}=c_{\max }\left(I_{j}\right)$, resides inside $\left[I_{j-1}, I_{j}\right]$. In this case, under linear interpolation, it can be shown that the maximum value of $V_{i, j(i, n+1)}^{n}$ is achieved when $\mathcal{I}_{j(i, n+1)}^{n}$ resides at $I_{j}$ or at its maximum/minimum bound. Thus, as $\Delta \tau^{n} \rightarrow 0$, the optimal value for $c$ is of bang-bang type, residing either at $c_{\max }\left(I_{j}\right), c_{\min }\left(I_{j}\right)$, or at zero.

Based on the above analysis, we can force the controls in (C.1) to be of bang-bang type by only examining controls $\zeta_{i, j}^{n+1}, \zeta_{i, j}^{n}$ that satisfy

$$
\zeta_{i, j}^{n+1} \in\left\{c_{\max }\left(I_{j}\right), c_{\min }\left(I_{j}\right), 0\right\} \quad ; \quad \zeta_{i, j}^{n} \in\left\{c_{\max }\left(\mathcal{I}_{j(i, n+1)}^{n}\right), c_{\min }\left(\mathcal{I}_{j(i, n+1)}^{n}\right), 0\right\} .
$$

Note that the controls from (C.8) may not be admissible ${ }^{4}$, that is, the resulting $\mathcal{I}_{j(i, n+1)}^{n}$ calculated from (C.2) either lies outside of domain $\left[0, I_{\max }\right]$ or does not exist.

\footnotetext{
${ }^{4}$ However, according to Lemma D.1, $\left(\zeta_{i, j}^{n+1}, \zeta_{i, j}^{n}\right)$ are always admissible if $\Delta \tau^{n}$ is sufficiently small.
} 
Taking the admissible control requirement into consideration, our bang-bang control method is summarized as follows: for each pair $\left(\zeta_{i, j}^{n+1}, \zeta_{i, j}^{n}\right)$ from (C.8), if it is admissible, then we evaluate the objective function in (C.1) using the pair and save the evaluated value as a candidate solution for problem (C.1).

Otherwise, assume that a pair $\left(\zeta_{i, j}^{n+1}, \zeta_{i, j}^{n}\right)$ satisfying (C.8) is not admissible, we evaluate the objective function in (C.1) using admissible controls that reside in the same region as the pair $\left(\zeta_{i, j}^{n+1}, \zeta_{i, j}^{n}\right)$ and result in the maximum or minimum bounds of $\mathcal{I}_{j(i, n+1)}^{n}$. To illustrate the idea, let us consider a specific case when $\left(\zeta_{i, j}^{n+1}, \zeta_{i, j}^{n}\right)=\left(c_{\max }\left(I_{j}\right), c_{\max }\left(\mathcal{I}_{j(i, n+1)}^{n}\right)\right)$ and $\left(\zeta_{i, j}^{n+1}, \zeta_{i, j}^{n}\right)$ is not admissible. In this case, the pair $\left(\zeta_{i, j}^{n+1}, \zeta_{i, j}^{n}\right)$ resides in region $\left[0, c_{\max }\left(I_{j}\right)\right] \times\left[0, c_{\max }\left(I_{j(i, n+1)}^{n}\right)\right]$. As explained above in the no bang-bang method, for all controls in this region, the corresponding values of $\mathcal{I}_{j(i, n+1)}^{n}$ consist of an interval $\left[I_{\text {min }}^{n}, I_{\text {max }}^{n}\right]$. Then we compute the value of the objective function in (C.1) by using two pairs of admissible controls that result in $\mathcal{I}_{j(i, n+1)}^{n}=I_{\min }^{n}$ and $\mathcal{I}_{j(i, n+1)}^{n}=I_{\max }^{n}$, respectively. A similar strategy is applied if other pairs of controls satisfying (C.8) are not admissible.

After evaluating the objective function in (C.1) using different pairs of admissible controls, as described above, we return the maximum value as the solution to problem (C.1).

\section{Proof for Lemma 4.4}

Prior to presenting the proof for Lemma 4.4, we first give the following Lemmas.

Lemma D.1. Let $C_{j}^{n+1} \subseteq C\left(\mathcal{I}_{j(i, n+1)}^{n}\right) \times C\left(I_{j}\right)$ be the set of admissible controls such that $\mathcal{I}_{j(i, n+1)}^{n}$ calculated from

$$
\mathcal{I}_{j(i, n+1)}^{n}=I_{j}-(1-\theta) \Delta \tau^{n}\left(\zeta_{i, j}^{n+1}+a\left(\zeta_{i, j}^{n+1}\right)\right)-\theta \Delta \tau^{n}\left(\zeta_{i, j}^{n}+a\left(\zeta_{i, j}^{n}\right)\right)
$$

is bounded within $\left[0, I_{\max }\right]$, where $C\left(\mathcal{I}_{j(i, n+1)}^{n}\right), C\left(I_{j}\right)$ are defined based on equation (2.7). Then by taking $\Delta \tau^{n}$ sufficiently small, we have

$$
C_{j}^{n+1}=C\left(\mathcal{I}_{j(i, n+1)}^{n}\right) \times C\left(I_{j}\right)
$$

provided that $c_{\max }, c_{\min }$ and $a(c)$ satisfy equations (2.1), (2.3) and (2.5), respectively.

Proof. Consider the case where $\epsilon^{*} \leq I_{j} \leq I_{\max }-\epsilon^{*}$, where $\epsilon^{*} \ll I_{\max }$ is independent of $h_{\min }$. In other words, we exclude a small strip of finite size near $I=0$ and $I=I_{\max }$.

If we let $\zeta_{i, j}^{n}, \zeta_{i, j}^{n+1}$ be two arbitrary controls satisfying

$$
\zeta_{i, j}^{n} \in C\left(\mathcal{I}_{j(i, n+1)}^{n}\right) \quad ; \quad \zeta_{i, j}^{n+1} \in C\left(I_{j}\right)
$$

then since $\zeta_{i, j}^{n}, \zeta_{i, j}^{n+1}$ are bounded, there exists a constant $D>0$ such that

$$
\left|\zeta_{i, j}^{n}+a\left(\zeta_{i, j}^{n}\right)\right|<D \quad ; \quad\left|\zeta_{i, j}^{n+1}+a\left(\zeta_{i, j}^{n+1}\right)\right|<D .
$$

Equations (D.1) and (D.4) imply that

$$
I_{j}-\Delta \tau^{n} D<\mathcal{I}_{j(i, n+1)}^{n}<I_{j}+\Delta \tau^{n} D .
$$


Thus, by taking $\Delta \tau^{n}<\min \left\{\frac{I_{j}}{D}, \frac{I_{\max }-I_{j}}{D}\right\}$, from equation (D.5), we obtain that $\mathcal{I}_{j(i, n+1)}^{n} \in\left[0, I_{\max }\right]$, assuming $\epsilon^{*} \leq I_{j} \leq I_{\max }-\epsilon^{*}$. The above argument shows that $\zeta_{i, j}^{n}$ and $\zeta_{i, j}^{n+1}$ are admissible controls such that $\left(\zeta_{i, j}^{n}, \zeta_{i, j}^{n+1}\right) \in C_{j}^{n+1}$ for a sufficiently small $\Delta \tau^{n}$. This amounts to the identities in (D.2) for the case $\epsilon^{*} \leq I_{j} \leq I_{\max }-\epsilon^{*}$.

Now, consider the nodes in the strip $I_{j}<\epsilon^{*}$. For $j$ fixed, we have $I_{j}=O\left(h_{\text {min }}\right)$, hence the condition on $\Delta \tau^{n}$ becomes

$$
\Delta \tau^{n} \leq \text { const. } \frac{h_{\min }}{D}
$$

which implies that we have consistency only if there is a condition on $(\Delta \tau) / h_{\min }$. Recall that we have assumed only that condition (3.24) holds for any constants $C_{3}, C_{4}$. Condition (D.6) adds an extra condition which seems unnatural. The strip $I_{j}>I_{\max }-\epsilon^{*}$ also gives rise to a similar condition.

In fact, this condition disappears if we carry out a more precise analysis. This proof is very long and tedious, and involves examination of many different cases. We will outline the proof for a simple case only.

Consider the fully implicit case, and $I_{j}<\epsilon^{*}$. In this case we have

$$
\mathcal{I}_{j(i, n+1)}^{n}=I_{j}-\Delta \tau^{n}\left(\zeta_{i, j}^{n+1}+a\left(\zeta_{i, j}^{n+1}\right)\right) .
$$

Equations (2.1), (2.5) and (2.6) imply that

$$
I_{j}-\Delta \tau^{n} c_{\max }\left(I_{j}\right)<\mathcal{I}_{j(i, n+1)}^{n}
$$

so that in order to have $\mathcal{I}_{j(i, n+1)}^{n}>0$, we must have

$$
\Delta \tau^{n} c_{\max }\left(I_{j}\right)<I_{j}
$$

From equation (2.1), we have that

$$
c_{\max }(I)=O(\sqrt{I}) ; I \rightarrow 0 .
$$

Assuming that $I_{j}=O\left(h_{\min }\right)$, then equations (D.9-D.10) give

$$
\Delta \tau^{n}=O\left(\sqrt{h_{\min }}\right)
$$

but this is a weaker condition than the assumption (3.24). Hence condition (D.9) is satisfied for any $\zeta_{i, j}^{n+1} \in C\left(I_{j}\right)$ when $\Delta \tau^{n}$ sufficiently small, with no additional restriction on $\Delta \tau^{n} / h_{\min }$ (other than assumption (3.24)). Equation (D.2) holds in this case. Similar arguments show that (D.2) holds in all cases for $0 \leq I_{j} \leq I_{\max }$, for $\Delta \tau^{n}$ sufficiently small, provided that $c_{\max }, c_{\min }$ and $a(c)$ satisfy equations (2.1), (2.3) and (2.5), respectively.

Lemma D.2. Suppose $F\left(c_{1}, c_{2}\right)$ and $H\left(c_{1}, c_{2}\right)$ are bounded functions defined in some domain $c_{1} \in$ $C_{1}, c_{2} \in C_{2}$. Then there exists a bounded function $Q\left(h_{\max }\right)$ where

$$
Q\left(h_{\max }\right) \leq \max _{c_{1}, c_{2}}\left|H\left(c_{1}, c_{2}\right)\right|
$$

such that

$$
\min _{c_{1}, c_{2}}\left\{F\left(c_{1}, c_{2}\right)+H\left(c_{1}, c_{2}\right) h_{\max }\right\}=\min _{c_{1}, c_{2}}\left\{F\left(c_{1}, c_{2}\right)\right\}+h_{\max } Q\left(h_{\max }\right),
$$


Proof. According to [22], we have that

$\left.\min _{c_{1}, c_{2}}\left\{H\left(c_{1}, c_{2}\right) h_{\max }\right\} \leq \min _{c_{1}, c_{2}}\left\{F\left(c_{1}, c_{2}\right)+H\left(c_{1}, c_{2}\right) h_{\max }\right\}-\min _{c_{1}, c_{2}}\left\{F\left(c_{1}, c_{2}\right)\right\} \leq \max _{c_{1}, c_{2}}\left\{H\left(c_{1}, c_{2}\right) h_{\max }\right)\right\}$.

Equation (D.13) directly follows from the above inequalities.

After presenting Lemmas D.1 and D.2, in the following we prove Lemma 4.4. We define vector $\phi^{n}$ with components $\left[\phi^{n}\right]_{i, j}=\phi\left(P_{i}, I_{j}, \tau^{n}\right)$. We first consider the discrete function $G_{i, j}^{n+1}$ in equation (3.25). Assuming the discretization in Appendix A is used, then from Taylor series expansions, we obtain that

$$
\left(L \phi^{n+1}\right)_{i, j}=(\mathcal{L} \phi)_{i, j}^{n+1}+O\left(\Delta P_{\max }\right) .
$$

Let us assume that the interpolation error in equation (3.20), due to operation $\Phi^{n+1}$, is $O\left(\left(\Delta I_{\max }\right)^{2}\right)$. In other words, linear or higher order interpolation is used to compute $\phi\left(P_{i}, \mathcal{I}_{j(i, n+1)}^{n}, \tau^{n}\right)$. Consequently, we can write

$$
\begin{aligned}
{\left[\Phi^{n+1} \phi^{n}\right]_{i, j} } & =\phi\left(P_{i}, \mathcal{I}_{j(i, n+1)}^{n}, \tau^{n}\right)+O\left(\left(\Delta I_{\max }\right)^{2}\right) \\
{\left[\Phi^{n+1} L \phi^{n}\right]_{i, j} } & =(\mathcal{L} \phi)\left(P_{i}, \mathcal{I}_{j(i, n+1)}^{n}, \tau^{n}\right)+O\left(\Delta P_{\max }+\left(\Delta I_{\max }\right)^{2}\right),
\end{aligned}
$$

where

$$
\mathcal{I}_{j(i, n+1)}^{n}=I_{j}-(1-\theta) \Delta \tau^{n}\left(\zeta_{i, j}^{n+1}+a\left(\zeta_{i, j}^{n+1}\right)\right)-\theta \Delta \tau^{n}\left(\zeta_{i, j}^{n}+a\left(\zeta_{i, j}^{n}\right)\right),
$$

and $\mathcal{L} \phi$ is regarded as a single function. In the above equation (D.17), we use (D.15) to estimate the error between the discrete operation $\left[L \phi^{n}\right]_{i j}$, as given in (3.19), and the exact operation $(\mathcal{L} \phi)_{i, j}^{n}$.

According to (D.18) and (D.16), we have that

$$
\begin{aligned}
\frac{\phi_{i, j}^{n+1}-\left[\Phi^{n+1} \phi^{n}\right]_{i, j}}{\Delta \tau^{n}}= & \left(\phi_{\tau}\right)_{i, j}^{n+1}+(1-\theta)\left(\zeta_{i, j}^{n+1}+a\left(\zeta_{i, j}^{n+1}\right)\right)\left(\phi_{I}\right)_{i, j}^{n}+\theta\left(\zeta_{i, j}^{n}+a\left(\zeta_{i, j}^{n}\right)\right)\left(\phi_{I}\right)_{i, j}^{n} \\
& +O\left(\Delta \tau_{\max }+\left(\Delta I_{\max }\right)^{2} / \Delta \tau_{\min }\right),
\end{aligned}
$$

where Taylor series is used to expand $\phi\left(P_{i}, \mathcal{I}_{j(i, n+1)}^{n}, \tau^{n}\right)$ at $\left(P_{i}, I_{j}, \tau^{n}\right)$. Similarly, expanding (D.17) at $\left(P_{i}, I_{j}, \tau^{n+1}\right)$ gives

$$
\left[\Phi^{n+1} L \phi^{n}\right]_{i, j}=(\mathcal{L} \phi)_{i, j}^{n+1}+O\left(\Delta \tau_{\max }+\Delta P_{\max }+\left(\Delta I_{\max }\right)^{2}\right)
$$

For convenience, we define

$$
W\left(P_{i}, I_{j}, \tau^{n+1} ; c\right)=\left(\phi_{\tau}+(c+a(c)) \phi_{I}-(c-a(c)) P-\mathcal{L} \phi\right)_{i, j}^{n+1} .
$$

Substituting equations (D.15), (D.19-D.20) and $\left(\phi_{I}\right)_{i, j}^{n}=\left(\phi_{I}\right)_{i, j}^{n+1}+O\left(\Delta \tau_{\max }\right)$ into $G_{i, j}^{n+1}$ given in (3.25), then writing $G_{i, j}^{n+1}$ in terms of notation (D.21), gives

$$
\begin{aligned}
& G_{i, j}^{n+1}\left(h_{\max }, \phi_{i, j}^{n+1},\left\{\phi_{k, j}^{n+1}\right\}_{k \neq i},\left\{\phi_{i, j}^{n}\right\}\right) \\
& =\min _{\left(\zeta_{i, j}^{n}, \zeta_{i, j}^{n+1}\right) \in C_{j}^{n+1}}\left\{(1-\theta) W\left(P_{i}, I_{j}, \tau^{n+1} ; \zeta_{i, j}^{n+1}\right)+\theta W\left(P_{i}, I_{j}, \tau^{n+1} ; \zeta_{i, j}^{n}\right)\right. \\
& \left.\quad+O\left(\Delta \tau_{\max }+\Delta P_{\max }+\left(\Delta I_{\max }\right)^{2} / \Delta \tau_{\min }+\left(\Delta I_{\max }\right)^{2}\right)\right\} \\
& \quad+\min _{\left(\zeta_{i, j}^{n}, \zeta_{i, j}^{n+1}\right) \in C_{j}^{n+1}}\left\{(1-\theta) W\left(P_{i}, I_{j}, \tau^{n+1} ; \zeta_{i, j}^{n+1}\right)+\theta W\left(P_{i}, I_{j}, \tau^{n+1} ; \zeta_{i, j}^{n}\right)+O\left(h_{\max }\right)\right\},
\end{aligned}
$$


where we use equations (3.24) to unify the mesh size/timestep size using $h_{\max }$. Note that the constant for $O$-notation in equation (D.22) is a function of $\zeta_{i, j}^{n}$ and $\zeta_{i, j}^{n+1}$. In other words, we may write $O\left(h_{\max }\right)=H\left(\zeta_{i, j}^{n}, \zeta_{i, j}^{n+1}\right) h_{\max }$, where $H$ is a bounded function of $\zeta_{i, j}^{n}, \zeta_{i, j}^{n+1}$.

According to Lemma D.1, by taking $\Delta \tau^{n}$ sufficiently small, given equations (2.1), (2.3) and (2.5), we can relax the constraint $\left(\zeta_{i, j}^{n}, \zeta_{i, j}^{n+1}\right) \in C_{j}^{n+1}$ in above equation (D.22) to $\zeta_{i, j}^{n+1} \in C\left(I_{j}\right)$, $\zeta_{i, j}^{n} \in C\left(\mathcal{I}_{j(i, n+1)}^{n}\right)$.

The above argument together with Lemma D.2 allows us to write equation (D.22) as

$$
\begin{aligned}
& G_{i, j}^{n+1}\left(h_{\max }, \phi_{i, j}^{n+1},\left\{\phi_{k, j}^{n+1}\right\}_{k \neq i},\left\{\phi_{i, j}^{n}\right\}\right) \\
& =(1-\theta) \min _{\zeta_{i, j}^{n+1} \in C\left(I_{j}\right)}\left\{W\left(P_{i}, I_{j}, \tau^{n+1} ; \zeta_{i, j}^{n+1}\right)\right\}+\theta \min _{\zeta_{i, j}^{n} \in C\left(\mathcal{I}_{j(i, n+1)}^{n}\right)}\left\{W\left(P_{i}, I_{j}, \tau^{n+1} ; \zeta_{i, j}^{n}\right)\right\}+Q\left(h_{\max }\right) h_{\max },
\end{aligned}
$$

where $Q$ is a bounded function satisfying (for some function $H\left(\zeta_{i, j}^{n}, \zeta_{i, j}^{n+1}\right)$ )

$$
Q\left(h_{\max }\right) \leq \max _{\zeta_{i, j}^{n} \in C\left(\mathcal{I}_{j(i, n+1)}^{n}\right), \zeta_{i, j}^{n+1} \in C\left(I_{j}\right)}\left|H\left(\zeta_{i, j}^{n}, \zeta_{i, j}^{n+1}\right)\right| .
$$

By (D.23), the left hand side of the equation (4.7) can be written using the notation (D.21) as

$$
\begin{aligned}
& \lim _{h_{\max } \rightarrow 0} \mid \min _{c \in C\left(I_{j}\right)}\left\{W\left(P_{i}, I_{j}, \tau^{n+1} ; c\right)\right\}-(1-\theta) \min _{\zeta_{i, j}^{n+1} \in C\left(I_{j}\right)}\left\{W\left(P_{i}, I_{j}, \tau^{n+1} ; \zeta_{i, j}^{n+1}\right)\right\} \\
& \quad-\theta \min _{\zeta_{i, j}^{n} \in C\left(\mathcal{I}_{j(i, n+1)}^{n}\right)}\left\{W\left(P_{i}, I_{j}, \tau^{n+1} ; \zeta_{i, j}^{n}\right)\right\}-Q h_{\max } \mid \\
& =\lim _{h_{\max } \rightarrow 0}\left|\theta \min _{c \in C\left(I_{j}\right)}\left\{W\left(P_{i}, I_{j}, \tau^{n+1} ; c\right)\right\}-\theta \min _{\zeta_{i, j}^{n} \in C\left(\mathcal{I}_{j(i, n+1)}^{n}\right)}\left\{W\left(P_{i}, I_{j}, \tau^{n+1} ; \zeta_{i, j}^{n}\right)\right\}-Q h_{\max }\right| \\
& =0,
\end{aligned}
$$

where the last equality follows because as $h_{\max } \rightarrow 0$, then $\Delta \tau^{n} \rightarrow 0$, and hence $C\left(\mathcal{I}_{j(i, n+1)}^{n}\right) \rightarrow$ $C\left(I_{j}\right)$. Therefore, we have proved the consistency of semi-Lagrangian discretization (3.25) according to Definition 4.3.

\section{References}

[1] H. Ahn, A. Danilova, and G. Swindle. Storing arb. Wilmott Magazine, 1, 2002.

[2] G. Barles. Convergence of numerical schemes for degenerate parabolic equations arising in finance. In L. C. G. Rogers and D. Talay, editors, Numerical methods in finance, pages 1-21. Cambridge University Press, Cambridge, 1997.

[3] G. Barles. Nonlinear Neumann boundary conditions for quasilinear degenerate elliptic equations and applications. Journal of Differential Equations, 154:191-224, 1999. 
[4] G. Barles and J. Burdeau. The Dirichlet problem for semilinear second-order degenerate elliptic equations and applications to stochastic exit time control problems. Communications in Partial Differential Equations, 20:129-178, 1995.

[5] G. Barles and E. Jakobsen. Error bounds for monotone approximation schemes for parabolic Hamilton-Jacobi-Bellman equations. Working Paper, Norwegian University of Science and Technology, 2005.

[6] G. Barles and E. Rouy. A strong comparison result for the Bellman equation arising in stochastic exit time control problems and its applications. Communications in Partial Differential Equations, 23:1945-2033, 1998.

[7] G. Barles and P. E. Souganidis. Convergence of approximation schemes for fully nonlinear equations. Asymptotic Analysis, 4:271-283, 1991.

[8] C. Barrera-Esteve, F. Bergeret, C. Dossal, E. Gobet, A. Meziou, R. Munos, and D. ReboulSalze. Numerical methods for the pricing of swing options: a stochastic control approach. Methodology and Computing in Applied Probability, 2006. to appear.

[9] R. Bermejo. Analysis of a class of quasi-monotone and conservative semi-Lagrangian advection schemes. Numerische Mathematik, 87:597-623, 2001.

[10] M. Bourgoing. Viscosity solutions of fully nonlinear second order parabolic equations with $l^{1}$ dependence in time and Neumann boundary conditions. Working paper, Université de Tours.

[11] R. Carmona and M. Ludkovski. Gas storage and supply guarantees: an optimal switching approach. Submitted to Management Science, 2005.

[12] S. Chaumont. A strong comparison result for viscosity solutions to Hamilton-Jacobi-Bellman equations with Dirichlet condition on a non-smooth boundary and application to parabolic problems. Working paper, Université Henri Poincaré I, May 2004.

[13] M. G. Crandall, H. Ishii, and P. L. Lions. User's guide to viscosity solutions of second order partial differential equations. Bulletin of the American Mathematical Society, 27:1-67, 1992.

[14] Y. d'Halluin, P. A. Forsyth, and G. Labahn. A penalty method for American options with jump diffusion processes. Numerische Mathematik, 97:321-352, 2004.

[15] Y. D'Halluin, P. A. Forsyth, and G. Labahn. A Semi-Lagrangian approach for American asian options under jump diffusion. SIAM Journal on Scientific Computing, 27(1):315-345, 2005.

[16] Y. d'Halluin, P.A. Forsyth, and K.R. Vetzal. Robust numerical methods for contingent claims under jump diffusion processes. IMA Journal of Numerical Analysis, 25:65-92, 2005.

[17] A. Dixit and R. Pindyck. Investment Under Uncertainty. Princeton University Press, Princeton, 1994.

[18] J. Douglas, Jr. and T. F. Russell. Numerical methods for convection-dominated diffusion problems based on combining the method of characteristics with finite element or finite difference procedures. SIAM Journal on Numerical Analysis, 19:871-885, 1982. 
[19] M. Falcone and R. Ferretti. Discrete time high-order schemes for viscosity solutions of Hamilton-Jacobi-Bellman equations. Numerische Mathematik, 67:315-344, 1994.

[20] M. Falcone and R. Ferretti. Convergence analysis for a class of high-order semi-Lagrangian advection schemes. SIAM Journal on Numerical Analysis, 35:909-940, 1998.

[21] W. H. Fleming and H. M. Soner. Controlled Markov processes and viscosity solutions. Springer, 2006.

[22] P. A. Forsyth and G. Labahn. Numerical methods for controlled Hamilton-Jacobi-Bellman PDEs in finance. Technical report, 2006. Working paper, University of Waterloo.

[23] P. Jaillet, E. I. Ronn, and S. Tompaidis. Valuation of commodity-based swing options. Management Science, 50(7):909-921, July 2004.

[24] E. R. Jakobsen. On the rate of convergence of approximation schemes for Bellman equations associated with optimal stopping time problems. Mathematical Models and Methods in Applied Science, 13:613-644, 2003.

[25] H. Li. Adaptive wavelet collocation methods for option pricing PDEs. PhD Thesis, University of Calgary, August 2006.

[26] O. A. Oleinik and E. V. Radkevic. Second order equations with nonnegative characteristic form. American Mathematical Society, Providence, 1973.

[27] H. Pham. On some recent aspects of stochastic control and their applications. Probability Surveys, 2:506-549, 2005.

[28] D. Pilopović. Energy Risk. McGraw-Hill, New York, 1998.

[29] O. Pironneau. On the transport diffusion algorithm and its applications to the Navier-Stokes equations. Numerische Mathematik, 38:309-332, 1982.

[30] D. M. Pooley, P. A. Forsyth, and K. R. Vetzal. Numerical convergence properties of option pricing PDEs with uncertain volatility. IMA Journal of Numerical Analysis, 23:241-267, 2003.

[31] R. Rannacher. Finite element solution of diffusion problems with irregular data. Numerische Mathematik, 43:309-327, 1984.

[32] M. Thompson, M. Davison, and H. Rasmussen. Natural gas storage valuation and optimization: a real options application. Working paper, University of Western Ontario, July 2003.

[33] M. Thompson, M. Davison, and H. Rasmussen. Valuation and optimal operation of electric power plants in competitive markets. Operations Research, 52(4):546-562, 2004.

[34] L. Triegeorgis. Real Options: Managerial Flexibility and Strategy for Resource Allocation. MIT Press, Boston, 1996.

[35] T. Ware. Swing options in a mean-reverting world. Presentation at: Stochastic Calculus and its applications to Quantitative Finance and Electrical Engineering, a conference in honour of Robert Elliott, Calgary, July 2005. 
[36] T. Ware and H. Li. Swing options with continuous exercise. Presentation at the Canadian Mathematical Society meeting, Calgary, June 2006.

[37] P. Wilmott. Derivatives: the theory and practice of financial engineering. John Wiley \& Sons, West Sussex, England, 1998.

[38] H. Windcliff, P. A. Forsyth, and K.R. Vetzal. Analysis of the stability of the linear boundary condition for the Black-Scholes equation. Journal of Computational Finance, 8(1):65-92, 2004.

[39] R. Zvan, P. A. Forsyth, and K. R. Vetzal. Discrete Asian barrier options. Journal of Computational Finance, 3:41-68, 1999. 